\title{
Flow Phenomena Leading to Surge in a Centrifugal Compressor
}

\author{
Bernhard Semlitsch*, Mihai Mihăescu \\ Linné FLOW Centre and Competence Center for Gas Exchange (CCGEx), Department of Mechanics, Royal Institute of Technology (KTH), \\ Osquars Backe 18, Stockholm, 10044, Sweden
}

\begin{abstract}
Surge is a global flow instability occurring in centrifugal compressors at low mass-flow rate operation. Due to its violent nature, it is the limiting factor for operability. To enhance the operating range, understanding of the flow instability inception when approaching surge is essential. Therefore, the flow evolution along a speed line is analysed by performing unsteady, three-dimensional flow simulations using a centrifugal compressor geometry with ported shroud. A stable operating condition, at high mass-flow rates, is compared to lower mass-flow rate operating conditions close to and at surge. The particularities of the flow-fields are analysed and described. A smooth flow-field is observed for the stable operating condition, whereas flow reversal manifesting as tip leakage at the outer periphery of the impeller occurs for all off-design operating conditions. The reversed flow exhibits swirling motion in the impeller rotation direction. This induces a globally swirling flow upstream of the impeller, which influences the flow incidence angles at the blades and hence, their efficiency. Proper orthogonal decomposition and dynamic mode decomposition have been performed to analyse the flow structures appearing with surge more thoroughly. For the lowest mass-flow rate operating condition, low frequency modes describing the filling and emptying processes during surge have been found.
\end{abstract}

Keywords: Compressor flows, Large Eddy Simulation, Flow decomposition methods, Turbocharged engines, Turbomachinery

\section{Introduction}

Engine downsizing has become a common practice to increase the specific efficiency and specific power of automotive internal combustion engines. In order to maintain the power output by recovering energy from the hot exhaust gases while reducing the displacement of the engine, turbocharging has become a widely applied technique. The reduction of the engine size leads to diminished thermal and frictional losses when compared to naturally aspirated internal combustion engines. Additionally the reduction of fuel required to achieve the same work results in less pollution production, which facilitates to comply with legislation emission restrictions. However, the recovered energy from the exhaust gases needs to blown off beyond the operational limits of the turbocharger and the engine efficiency is reduced. Wang et al. [1] analysed the impact of high altitude operation of a turbocharged engine and show that the lowered ambient pressure reduces the effectiveness of the

\footnotetext{
${ }^{*}$ Tel. +46 704742657

Email address: bernhard@mech.kth.se (Bernhard Semlitsch)
}

turbocharger due to a narrowing of the operation range. Hence, a wide turbocharger operating range is desired to boost the engine at all operating conditions.

The operating range and performance parameters governing the compressor characteristics, i.e. pressure ratio, mass-flow rate, and efficiency, are commonly described in a compressor specific operation map. The obtainable boost pressure is given as ratio of the total pressure $p_{0,2}$ at the compressor outlet to the total pressure $p_{0,1}$ at the compressor inlet and illustrated as function of mass-flow rate $\dot{m}$ and rotational speed $n$. Commonly, the adiabatic efficiency $\eta$ is utilised to quantify the compressor performance as a ratio of the ideally obtained work $W^{i s}$ to the actually supplied work $W^{a c t}$. The actually performed work is given by the rotational velocity $\omega$ and the torque $\tau$. The ideally obtained work is proportional to the temperature increase from the suction inlet to the compressor outlet, which leads to the expression [2];

$$
\eta=\frac{W^{i s}}{W^{a c t}}=\frac{\dot{m} c_{p}\left(T_{0,2}^{i s}-T_{0,1}\right)}{\tau \omega},
$$


where $c_{p}$ is the heat capacity at constant pressure, $T_{0}$ is the total temperature, and the indexes 1 and 2 are referencing the quantities to the inlet and outlet, respectively. The superscript $i s$ denotes that the quantity is estimated via isentropic relations. Since the compressor wheel is usually driven by a turbine extracting energy from hot exhaust gases and transonic flow conditions are reached in parts of the compressor map, it is more convenient and realistic to assess the actual temperatures instead of rotational velocity and torque. Therefore, the relation

$$
\eta=\frac{\dot{m} c_{p}\left(T_{0,2}^{i s}-T_{0,1}\right)}{\tau \omega}=\frac{\dot{m} c_{p}\left(T_{0,2}^{i s}-T_{0,1}\right)}{\dot{m} c_{p}\left(T_{0,2}-T_{0,1}\right)}
$$

is used in compressor operating maps. The ideal temperature rise results due to gas compression from the inlet total pressure $p_{0,1}$ to the outlet total pressure $p_{0,2}$, which can be estimated via isentropic relations,

$$
\begin{aligned}
\eta & =\frac{\dot{m} c_{p}\left(T_{0,2}^{i s}-T_{0,1}\right)}{\dot{m} c_{p}\left(T_{0,2}-T_{0,1}\right)}=\frac{T_{0,1}\left(\frac{T_{0,2}^{i s}}{T_{0,1}}-1\right)}{\left(T_{0,2}-T_{0,1}\right)}= \\
& =\frac{T_{0,1}\left(\frac{p_{0,2}}{p_{0,1}}-1\right)}{\left(T_{0,2}-T_{0,1}\right)}=\frac{{\frac{p_{0,2}}{p_{0,1}}}^{\frac{\gamma-1}{\gamma}}-1}{\frac{T_{0,2}}{T_{0,1}}-1},
\end{aligned}
$$

where $\gamma$ is the averaged ratio of specific heats between inlet and outlet. The required quantities (i.e. total temperature ratio and total pressure ratio) to estimate the compressor efficiency are obtained by measurements or simulations.

The operating range, outlined on the compressor map, is restricted by two limits called surge (at low mass-flow rates) and choke (at high mass-flow rates). At these limits, the efficiency of the compressor is lower than at design operating conditions. The surge phenomenon is a global flow instability affecting the entire compressor geometry, which can cause large vibratory stresses in the blades and lead to the breakdown of operability. The large vibrations induce a loud characteristic noise, which can become dominant. Hence, to operate the compressor into surge is not desired, since this could lead to fatigue. Therefore, the compressor is usually bypassed in operation conditions close to surge at the so-called surge line to prevent damage. The surge line criterion indicating the onset of surge is chosen by the manufacturer and provided to the costumer. Andersen et al. [3] discuss different definitions of this criterion utilised by individual fabricators. Due to the implied uncertainty with different surge margin definitions, internal combustion engine producer apply safety margins to the compressor operation map. Better understanding of the surge phenomenon and its cause would allow expanding the potential of the turbocharger in a wider range.

Surge occurs at low engine speeds combined with high loads, e.g. during rapid accelerations, high altitude operation, or gearshifts, and is basically a phenomenon of air stalling with partial flow reversal in the compressor. Globally induced mass-flow rate and pressure ratio oscillations characterise surge. Fink et al. [4] described these oscillations as emptying and refilling process of the piping tract volume downstream of the compressor, which governs frequency and amplitude of these oscillations. Galindo et al. [5] showed with experiments and numerical modelling that a larger volume downstream of the compressor can lead to deep destructive surge with low frequency, whereas a smaller volume can result in a high frequent gentle surge. At off-design conditions prior to surge, stall or rotating stall may occur. However, these phenomena restrict the compressor efficiency, but are not as savage as surge.

Many active and passive flow control strategies have been developed in order to increase the operation width of centrifugal compressors. The ported shroud is a simple and reliable passive flow control technique used to increase the surge margins by allowing some flow to recirculate from the blade passage back into the compressor inlet. Yang et al. [6] showed that flow unsteadiness is drained out of the blade passage at the blade tips and the flow streaming through the blade passages is stabilised. Due to the simplicity and reliability of this passive flow control device, the ported shroud is widely used in automotive turbocharger compressors. It is worthwhile to mention that induced swirling motion generated upstream of the compressor inlet against the wheel rotation was used by Galindo et al. [7] and Figurella et al. [8] to shift the surge limits by stabilising the flow at off-design operation conditions.

Analysis of the flow at surge operating conditions is necessary to understand the phenomenon. However, the experimental assessment of the flow-field in confined compressor geometries is challenging. Typically, pressure, mass-flow, and temperature point measurements in probes are performed. At higher effort, Laser Doppler Velocimetry (LDV) and Particle Image Velocimetry (PIV) flow measurements have been used to investigate the flow in a partial domain. With computational simulations, the entire flow-field in the compressor is available, but the numerical challenges are multifaceted. The flow velocities are typically high in automotive turbocharger compressors, close to transonic speeds. Compressibility effects might occur and the problem specific Reynolds number is relatively high. Further, the geometry of a turbocharger compressor 
is complex and the wheel is rotating with respect to the static housing. Therefore, the demand on the selected computational approach is high, which has to handle the two main issues, i.e. compressible turbulence modelling and treatment of the wheel rotation. Common approaches used for most accurate simulation of centrifugal compressors are either computationally resource demanding or involve advanced numerical modelling of the occurring phenomena. Sundström et al. [9] showed by comparing numerical predictions with experiments that steady-state Reynolds Averaged Navier-Stokes (RANS) simulations can be utilised to characterise the performance of the compressor at stable operating conditions. The rotation of the wheel with these kinds of computations is commonly handled by the steady-state frozen rotor approach. Thereby, the rotation of the wheel is modelled by using a rotating reference frame. At the interface between rotor and stator, the forces are converted to the other coordinate system. However, this method gives only the solution for one fixed reference position of the frozen rotor. The mixing plane approach models the continuous rotation of the wheel using circumferential averaging. Several improved methods, such as the Average-Passage Equation System (APES) method or the Non-Linear Harmonic (NLH) method, have been developed to improve the predictability when rotor stator interactions become important. However, towards surge the flow-field begins to oscillate globally and steady-state approaches cannot capture the physics of pulsating flow dynamics accurately. Hence, unsteady simulation approaches are required.

Unsteady RANS formulations [10], Detached Eddy Simulation (DES) [11] and the Large Eddy Simulation (LES) approach [12] have been used to describe the unsteady flow-field at off-design operating conditions. Unsteady RANS resolves the integral scales in a turbulent flow-field while all smaller flow scales are modelled. Bourgeois et al. [11] compared numerical simulation approaches showing that a blind choice of turbulence closures can lead to erroneous simulation results. The reliability and accuracy of these approaches are highly sensitive to the turbulence model employed and the selection of wall treatment.

Unlike RANS based formulations, LES solves the most energetic structures in the flow-field down to the inertial subrange while modelling only the unresolved dissipative scales. Therefore, the LES approach requires a finer mesh, which represents a higher computational effort. Especially, accurate prediction of boundary layer effects is numerically expensive, since fine clustering of cells at the walls is required to resolve near wall effects. However, many phenomena in the centrifugal compressor, including surge, are governed by large-scale flow structures rather than small structures generated nearby the walls. Hence, when large-scale phenomena are investigated, under-resolved wall resolutions in combination with modelling are commonly utilised for computational efficiency reasons, whereas the large flow structures are resolved as accurate as possible. Therefore, the zonal method DES, applying the RANS approach in the under-resolved domain and LES in the resolved domain region [11], or LES with wall functions [13] are preferably used. The effect of wall functions and detailed wall modelling has been investigated by Mendonca et al. [14] showing that the largescale flow structures are not affected by carefully employed wall treatment. Gourdain et al. [15] underline in a review of numerical methods for compressor flows the relevance of wall functions with expensive computations. Within the framework of unsteady flow simulations, several numerical techniques are available to handle the rotation of the wheel. The frozen rotor approach, previously mentioned, has also been used for unsteady simulations. However, a more accurate technique for this purpose is the sliding mesh approach. Two mesh grids, reassembling the stationary compressor components and the rotating impeller respectively, are used. During the rotation of the mesh, the numerical cell connectivity changes on either side of the common interface at each time step. The cell values are interpolated over the interface to the nearest joint cell. Therefore, the sliding mesh approach is numerically very expensive. Since the flow-field has to be interpolated from one interface side to the other, the numerical accuracy is slightly decreased and coherent flow structures may be altered with this process. However, this procedure with accurate interpolation provides the most accurate handling of the flow over the interface.

The unsteady flow motion is often assessed by statistical examination of quantities, e.g. in terms of mean and variance of gathered flow variables. However, these distributions do not sufficiently identify the spatial characteristics or the degree of temporal variation of flow instabilities. Therefore, flow decomposition methods have been commonly used to extract the coherent flow structure characteristics. The Proper Orthogonal Decomposition (POD) method has been successfully used in a wide range of applications to visualise energetic large-scale structures of the flow. Hence, unsteady flow motion can be investigated at a higher level and large coherent structures can be identified accordingly to their correlation. However, POD modes are not necessarily associated with one single frequency 
and therefore, this method is not ideal for spectral identification of flow features. The Dynamic Mode Decomposition (DMD) method provides unique modes corresponding to one frequency. Thus, this flow decomposition method is perfectly suited to investigate dynamic key features in flows associated with tonal characteristics, such as surge. Further, modal flow analysis exhibit the potential to describe more accurately the complex flow phenomena by ordinary differential equations, which can be included in one-dimensional engine simulation codes. Nakonieczny [16] developed such a onedimensional flow model for predicting entropy production in a turbocharged diesel engine and achieved a reasonable comparison with experiments. This model allows finding ideal combinations of engine setting, as e.g. valve lift curves. The validity of such codes can be extended towards off-design operating conditions by representing the complex flow phenomena in low-order simulations.

Steady-state RANS and one-dimensional simulations allow optimising the geometry in a wide operating range with numerous simulations at reasonable numerical effort and represent therefore the state of the art for geometry design of turbocharger compressors. Unsteady simulations are too expensive to calculate the performance at many operating points and are therefore only performed for research purposes at selected conditions. However, unsteady simulation approaches are required for the investigation of flow structures. Especially, for global flow instabilities affecting the entire compressor, accurate simulations capturing the flow features of the entire $360^{\circ}$ geometry are essential employing the sliding mesh technique. Such computationally expensive calculations with accurate turbulence treatment, as presented in the present work, are only performed by a few research groups at present time. We analyse in detail the flow-field evolution occurring in a centrifugal ported shroud compressor of an automotive turbocharger while decreasing progressively the mass-flow rate along a constant speed line, from a stable operating point towards an unstable lower massflow rate operating points. Although, many researchers have reported global characteristics of surge, the largescale flow structures occurring in the compressor have not been assessed. Understanding the large-scale flow structures better would allow to invent improved techniques for delaying surge. Therefore, we employ modal decomposition methods to characterise these flow structures leading to surge. Thereby, this study represents, the first modal flow decomposition analysis of the threedimensional flow in a centrifugal compressor.

\section{Case Setup}

The investigated ported shroud compressor geometry of an automotive turbocharger, the Honeywell GT40, is shown in Fig. 1 (a). The centrifugal compressor is designed for heavy-duty truck engines in a power range of 400 to $850 \mathrm{~kW}$. The wheel has 10 full blades with a back sweep angle of $25^{\circ}$ at the discharge. The impeller is designed with an exducer diameter of $88 \mathrm{~mm}$ and a trim ratio of 56. The compressor diffuser is vaneless with an exit radius of $152 \mathrm{~mm}$ and the diffuser area ratio is 0.57 . In order to extend the operating range, the compressor is equipped with a ported shroud, which allows a part of the possible back-flow occurring under unstable operating conditions to recirculate from the blade passage through the shroud cavities back into the main flow upstream of the impeller inlet. Figure 1 (b) illustrates the operating principle of the ported shroud at off-design operation conditions, where the temperature contours indicate recirculating fluid. The red arrow indicates the flow path for normal operation and the purple arrow sketches the recirculating flow path. The shroud is supported by four ribs, which are arranged in asymmetric manner to prevent amplification of harmonic oscillations causing vibrations and high cycle fatigue.

The evolution of the flow-field in the centrifugal compressor from stable operating conditions towards the surge phenomenon is investigated by analysing data considering five cases on a constant speed line of 64 $\mathrm{krpm}$. The operating conditions for each of these are listed in Table 1, where the employed boundary condition values have been obtained by Gancedo et al. [17] in an experimental investigation of the same compressor geometry. For all cases, the mass-flow rate and total temperature have been specified at the compressor inlet, where the total temperature was set to $296 \mathrm{~K}$. At the compressor exit, an outlet pipe has been attached to extend the numerical domain. At the outlet boundary, a constant static pressure has been imposed, which is specified in Table 1 . No-slip, adiabatic boundary conditions have been applied at the walls.

The simulations I and II were initialised with flow solutions of steady-state RANS calculations. The solutions towards surge were obtained by progressively reducing the mass-flow rate at the compressor inlet within the transient simulations. The data sampling was activated when statistical independence from the initial conditions was reached. 


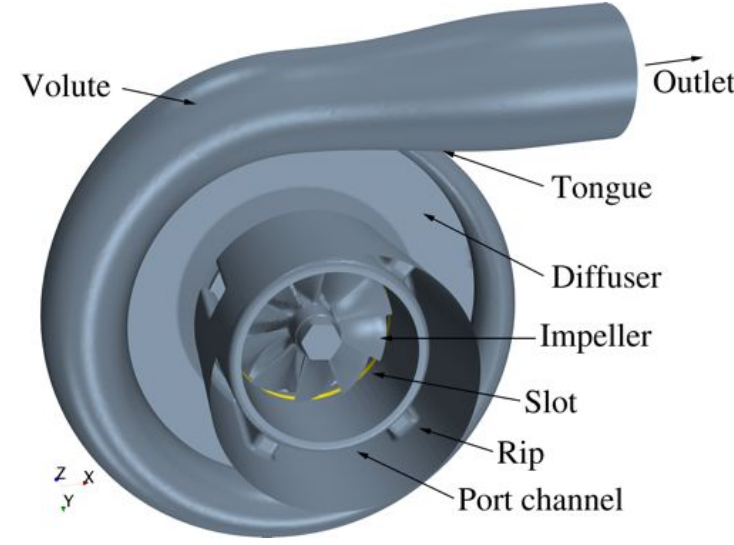

(a) geometry

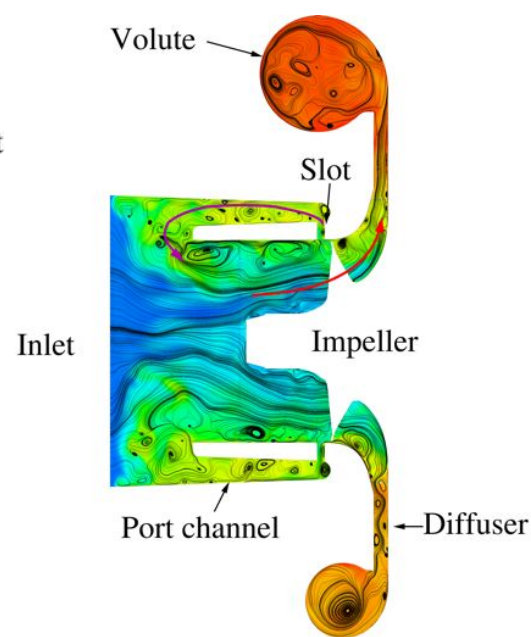

(b) mid-plane view

Figure 1: The geometry of the centrifugal compressor is illustrated to the left, where the axial location of the shroud bleeding slot is indicated with respect to the wheel by the surrounding yellow band. Figure (b) illustrates the working principle of the ported shroud during off-design operating conditions; the streamlines are shown on top of the temperature contours.

Table 1: The computed cases are listed with the applied boundary conditions, i.e. the mass-flow rate $\dot{m}$ and the static pressure at the outlet $p_{\text {exit }}$ provided by experimental measurements.

\begin{tabular}{ccc} 
case & $\dot{m}(\mathrm{~kg} / \mathrm{s})$ & $p_{\text {exit }}(\mathrm{kPa})$ \\
\hline I & 0.270 & 156 \\
II & 0.105 & 165 \\
III & 0.080 & 165 \\
IV & 0.065 & 165 \\
V & 0.050 & 165
\end{tabular}

\section{Numerical Method}

The numerical procedure is outlined by the flowchart provided in Fig. 2, where boundary conditions and simulation initialisation have been discussed in the previous section. The fundamental core of the simulation procedure is the numerical solving of the discretised conservation equation system. These conservation equations are the three-dimensional compressible Navier-Stokes equations, the mass conservation equation, and the energy conservation equation, which have been simulated with a compressible finite volume based solver, Star$\mathrm{CCM}+$. The ideal gas equation has been used to describe the material properties of the fluid air and to close the equation system.

The time is advanced at each outer iteration. For time integration, a second order accurate implicit scheme has been used, where a constant time-step of $1.3 \cdot 10^{-5} \mathrm{~s}$ has been applied for time advancement. Before the next outer iteration, the rotating domain revolves around the impeller axis. The handling of the wheel rotation has been performed using the sliding mesh technique.

Seven inner iterations are performed at each time-step to control the solution accuracy, where each time-step the solution has been converged approximately three orders of magnitude. The second order bounded central difference scheme was employed for the spatial terms in the equations. This scheme consists of a blend of a central difference and an upwinding scheme to guarantee boundedness of the solution and non-oscillatory behaviour of the discretisation scheme. The amount of upwinding introduced is controlled by the normalisedvariable diagram approach to stabilise the numerical scheme when necessary, where a maximum amount of upwinding was set to $10 \%$.

An unstructured polygonal mesh grid with a total amount of 6.3 million cells and a base size of $2 \mathrm{~mm}$ is used to discretise the complex compressor geometry, where the regions of interest (e.g. wall regions and regions of expected large gradients in the flow variables) have been refined. Prism layers next to the walls have been used with a grid stretching factor of 1.1. The $y+$ values lie between 0.8 and 21 . However, since the grid is not designed to resolve all the near wall regions, a wall function approach towards the walls has been used. Gourdain et al. [15] state that this range of wall resolution in combination with wall functions is appropriate for turbomachinery flow simulations. Further, the use 


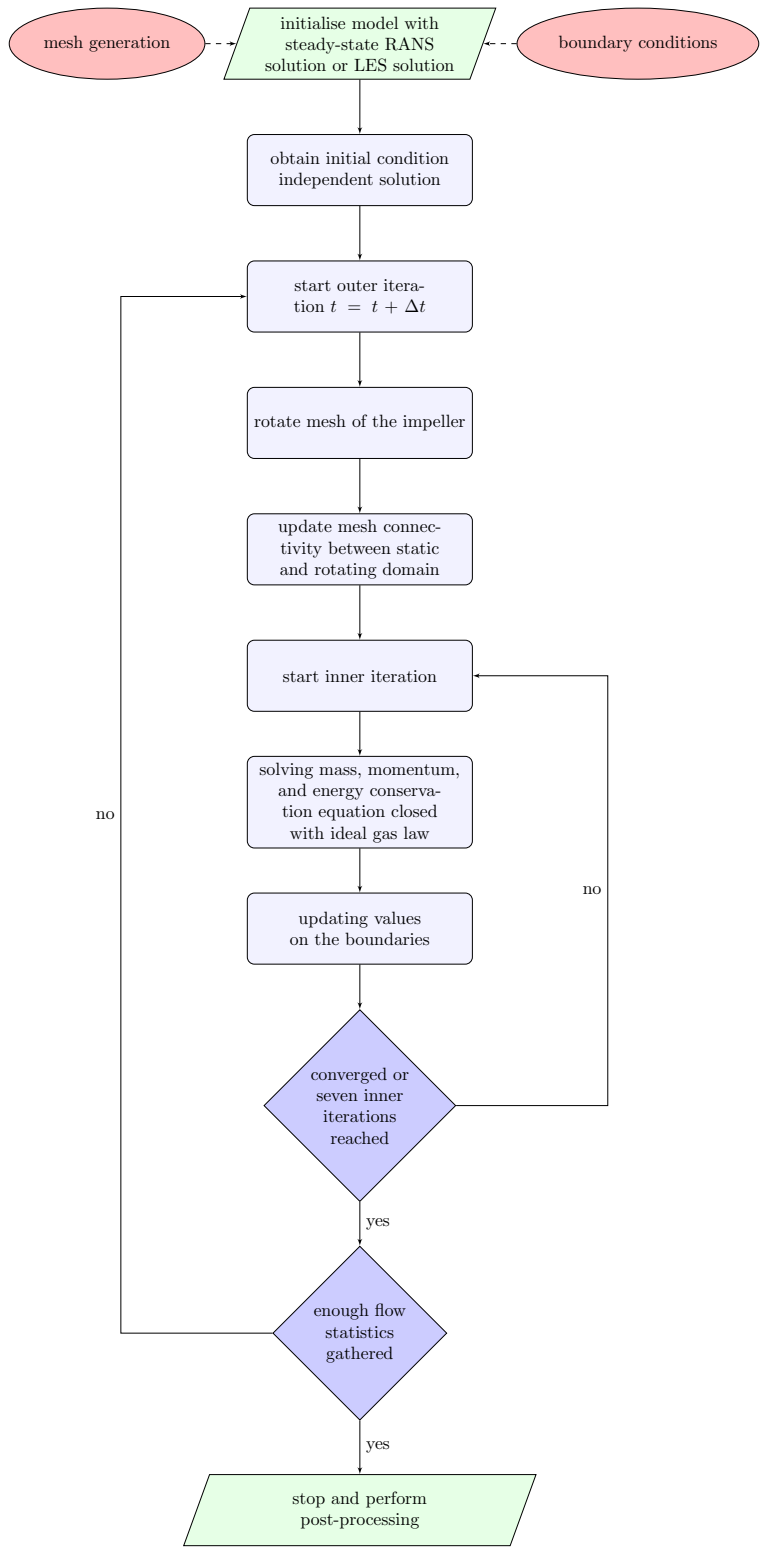

Figure 2: The numerical simulation procedure is illustrated.

of wall functions can be motivated since the flow instability growth in the boundary layer is slow and requires a long time to evolve compared to the instability growth in a shear layer formed due to geometrical constrains. Further, Sundström et al. [18] demonstrate that only $1 \%$ of the total net torque transmitted by the rotor to the flow evolves in viscous forces, while the substantial proportion induces pressure forces resulting in largescale flow motion. Hence, the flow in the compressor is governed by large-scale unsteady flow motion, which is dominated by the geometry and the boundary condi- tions applied.

Due to the high flow speed and gradients occurring in centrifugal compressors, turbulent flow motion is induced. The acceleration and compression of the flow in particular regions of the centrifugal compressor challenges turbulence treatment, because hardly any generally valid assumptions about the turbulence behaviour can be made. Since wrongly predicted dissipation of turbulence associated with modelling might alter the observed flow characteristics, an approach that involves the lowest possible amount of modelling to predict the global flow features is desired. With the LES approach, the numerical grid resolves the large characteristic flow scales and only the smallest scales are modelled. Due to the spatial filtering of the equations by the underresolved mesh grid, a sub-grid stress term representing the unresolved small scales adds to the governing equations. At the smallest flow scales, the kinetic energy is dissipated by molecular viscosity into heat. In the early 60s, Smagorinsky [19] developed a subgrid scale (SGS) model similar to the eddy-viscosity concept in the RANS framework, including artificial viscosity. The main purpose was to stabilise the numerical procedure for under-resolved simulations, since the missing dissipation at the level of the smallest, unresolved scales lead to unphysical oscillations of the flow solution. Since this SGS model was initially developed to prevent from numerical stability issues, the model lacks of physical reliability. Moreover, it is reliant on empirical calibration constants. However, it is today one of the most popular SGS model applied for problems involving turbulence in compressible flows.

The amount of kinetic energy, dissipated by the smallest flow scales, depends on the amount transferred from the large-scales to the small-scales through the energy cascade. Hence, an appropriate numerical discretisation scheme with low dissipative error, e.g. the central difference scheme, is desired for the convective terms to capture accurately the turbulence energy cascade. At a distance from boundaries, the small turbulent flow structures exhibit statistically a universal character. Hence, these scales can be modelled based on this assumption, e.g. by SGS models. However, the contribution of the subgrid scale models is commonly of the order of the truncation error of the numerical scheme [20], which requires explicit filtering of the governing equations to provide accuracy [21]. Thus, one can use the numerical truncation error to mimic the effect of the smallest turbulent scales. This approach is commonly known as monotone integrated LES or implicit LES and the mathematical foundation for this approach is described by Margolin and Rider [22]. Margolin et 
al. [23] demonstrate via mathematical derivations that the numerical truncation error terms can be interpreted as a Clark subgrid scale model for the momentum conservation equation and as a Smagorinsky-type subgrid scale model for the energy conservation equation, when a non-oscillatory finite volume discretisation of at least second order accuracy is used. Further, it is shown that the kinetic energy is absolutely decreasing. Fureby and Grinstein [24] demonstrate that a normalised-variable diagram discretisation scheme, as the currently used, is applicable for implicit LES. The remarkable properties of this approach lead to a subgrid scale model without calibration constants and without explicit filter. Due to the computational efficiency of implicit LES, this approach is used in the present investigation.

A comparison of numerical simulations with a similar setup, same compressor geometry, and experimental measurements has been shown previously by Semlitsch et al. [12]. The computational simulation results have been compared to global performance data (in terms of efficiency and compressor pressure ratio) and to PIV flow measurements at the shroud entry. A comparison of the numerical results to the experimental measurements by Gancedo et al. [17] in terms of the total pressure ratio and efficiency is also shown in Fig. 3 to illustrate the computed operating conditions with respect to the complete compressor characteristic along a constant speed line of $64 \mathrm{krpm}$. The mass-flow rate was continuously reduced from the highest mass-flow rate to the lowest in the experiments, while the pressure ratio and the compressor efficiency were measured. The same measurements have been performed from the lowest mass-flow rates to the highest. The difference in the results was less than $2 \%$. The observed differences between simulations and experiments can be related to the different modelling set-up. The gap between back plate and stator was not modelled in the numerical simulations, which has been shown to lead to an increase in predicted efficiency and pressure ratio [25]. Further, a rather short exit pipe has been attached to the compressor within the numerical simulations as compared with the experimental setup. Since the piping volume downstream of the compressor relates to the surge frequency and amplitude, it is expected that the numerical simulations and the experiments capture the pressure ratio drop for the deep surge condition differently. Further, one can observe in Fig. 3 that the pressure ratio fluctuations, indicated by error bars, are increasing significantly as the mass-flow rate reduces and the compressor approaches unstable operating conditions. However, the observed trends between the numerical and experimental results are in good agreement. For the experimental setup of the PIV measurements at the shroud entry, a bell mouth was mounted upstream of the compressor inlet. This was not simulated within the current LES calculations. To conclude, minor differences between the numerically and experimentally obtained compressor performance parameters and flow-fields are observed and overall a reasonable agreement between the PIV measurements and the numerical data at the shroud entry could be achieved, as shown by Semlitsch et al. [12].

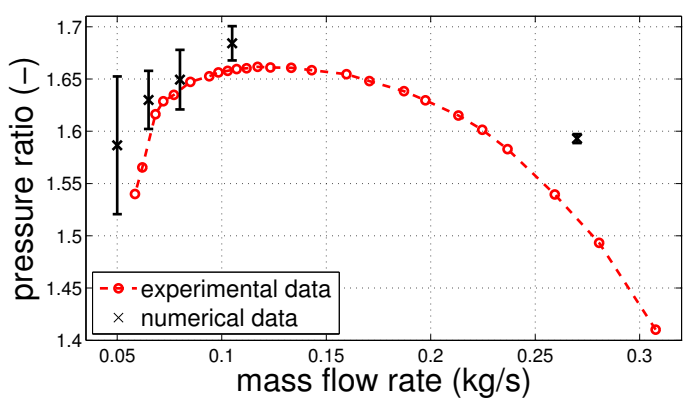

(a) Pressure ratio

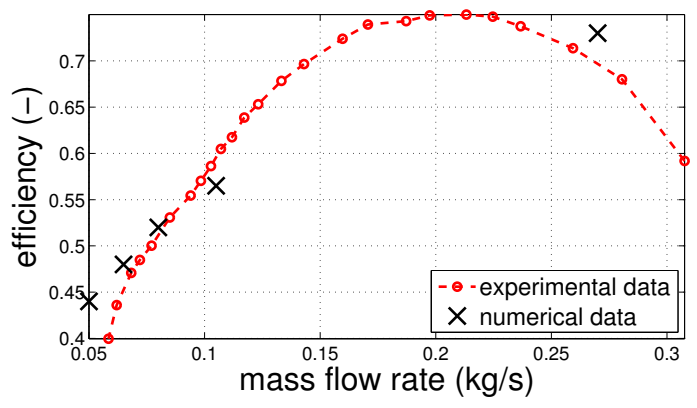

(b) Compressor efficiency

Figure 3: The computed cases are marked on the experimentally measured compressor map for the speed line of $64 \mathrm{krpm}$. Error bars for the numerical results indicate the variance of the pressure ratio.

A grid resolution study for the same compressor geometry using a similar numerical setup has been performed by Sundström et al. [18]. Only minor differences $(<1 \%)$ in terms of the global performance parameters have been observed with grid size variation. Further, line plot comparison revealed that the flow solution was altered insignificantly with increased mesh resolution and the same trends as for the experimental pressure measurement data has been captured. The monitored probe signal spectra for the three different mesh resolutions have been contrasted with experimental point measurements. The comparison showed that with the resolution used, fluctuations below $20 \mathrm{kHz}$ are accurately resolved. 


\section{Results}

In this section, the results of the LES calculations are presented, where the focus is held on the high massflow rate case $\dot{m}=0.27 \mathrm{~kg} / \mathrm{s}$, the onset of surge case $\dot{m}=0.105 \mathrm{~kg} / \mathrm{s}$, and the lowest mass-flow rate case studied $\dot{m}=0.05 \mathrm{~kg} / \mathrm{s}$. The flow-fields predicted for operating conditions in between the near surge case $(\dot{m}=0.105 \mathrm{~kg} / \mathrm{s})$ and the lowest mass-flow rate case $(\dot{m}=0.05 \mathrm{~kg} / \mathrm{s})$ do not exhibit large changes in the flow-field and follow the general trends. The high massflow rate case $\dot{m}=0.27 \mathrm{~kg} / \mathrm{s}$ has been simulated over 720 revolutions, the near surge case $\dot{m}=0.105 \mathrm{~kg} / \mathrm{s}$ has been computed over approximately 375 revolutions, and the simulation of the lowest mass-flow rate case $\dot{m}=0.05 \mathrm{~kg} / \mathrm{s}$ was run over 600 revolutions.

The evolution of the flow-field in the diffuser and volute with a progressive reduction of the mass-flow rate is illustrated in Fig. 4 and Fig. 5 in terms of the timeaveraged velocity magnitude and time-averaged static pressure, respectively. For the stable operating condition with a mass-flow rate of $\dot{m}=0.27 \mathrm{~kg} / \mathrm{s}$, the flow separates from the walls at the entrance into the main shroud and there, a separation bubble forms (Fig. 4 (a)). Higher velocity magnitudes can be observed as compared with the lower mass-flow rate cases. Even for this stable operating condition, flow recirculates through the shroud cavity back to the compressor inlet and the tip leakage is cleared. The time-averaged velocity magnitude contours in the diffuser and volute are decaying smoothly in radial direction. High velocity regions at the backside of the blades can be noticed (Fig. 4 (a)). A small low velocity region in the conical exit diffuser of the volute can be observed, which results from the strongly swirling flow in the volute. The corresponding static pressure contours, shown in Fig. 5 (a), reveal lowpressure regions at the blade tips. The static pressure increases continuously in radial direction of the diffuser. Especially, a high static pressure region can be observed at the outer periphery of the volute, which is initiated under the compressor tongue and evolves in flow direction until the six o'clock location is reached.

As shown in Figs. 4 (b) and (e), and Fig. 5 (b), the flow-field changes drastically when the mass-flow rate is reduced to $\dot{m}=0.105 \mathrm{~kg} / \mathrm{s}$. High velocity magnitudes reaching out of the shroud cavity indicate the amplified recirculating flow for this operating condition. Additionally, the velocity magnitudes in the shroud cavity are considerably increased. Jet-like structures develop from the recirculating flow coming out of the shroud cavity, which are bent back into the main shroud, as previously shown by Semlitsch et al. [12]. The unsteady flow struc- tures are notable far downstream into the diffuser region and the structures are responsible for a slight peak of about $250 \mathrm{~Hz}$ and $500 \mathrm{~Hz}$ in the frequency spectra. The spectral properties of the flow are discussed in a later section.

The low velocity zone manifesting due to the flow separation at the main shroud inlet, which has been visible for the high mass-flow rate operating condition, is not observable for the mass-flow rate case of $\dot{m}=0.105$ $\mathrm{kg} / \mathrm{s}$. Moreover, the high velocity magnitude regions at the outer periphery of the main shroud evolve from far downstream at the impeller discharge. These highlight the high velocity magnitudes of the back-flow nearby the wall. At the blade tips, the high velocity regions observed for the stable operating condition case in Fig. 4 (d) are highly reduced notable in Fig. 4 (e), although the rotating speed of the wheel is unaltered. A high velocity zone establishes in the near region of the impeller facing the volute tongue, which was not visible for the stable, high mass-flow rate case. In this region, the flow velocities are higher than for the high mass-flow rate case. However, in the rest of the volute, the flow velocities are low compared to the high mass-flow rate case. Further, boundary layer development can hardly be observed for the stable $\dot{m}=0.27 \mathrm{~kg} / \mathrm{s}$ condition, whereas a significant reduction of flow velocity nearby the diffuser walls is visible for the operating condition $\dot{m}=0.105$ $\mathrm{kg} / \mathrm{s}$. The time-averaged static pressure, shown in Fig. 5 (c), exhibits that the compressor pushes against a higher static pressure and enhanced gradients in the radial directions can be noted as compared with the high massflow rate case. A strong negative pressure gradient from the volute tongue towards the two o'clock location is observable. That is where the low-pressure region establishes towards the two o'clock location under the compressor's tongue. The static pressure rises continuously from the two o'clock location in clockwise direction towards the compressor outlet. Further, the time-averaged static pressure distribution in the blade passages exhibits the lowest values within the blade passages at the inner backside of the blades, whereas for the high mass-flow rate case, the time-averaged static pressure is lowest at the blade tips.

By reducing the mass-flow rate even further from $\dot{m}=$ $0.105 \mathrm{~kg} / \mathrm{s}$ to $\dot{m}=0.05 \mathrm{~kg} / \mathrm{s}$, the abnormalities in the flow and pressure fields observed at the mass-flow rate of $\dot{m}=0.105 \mathrm{~kg} / \mathrm{s}$ and the deviations from the stable situation (e.g. $\dot{m}=0.27 \mathrm{~kg} / \mathrm{s}$ ) are enhanced. At the operating condition with $\dot{m}=0.05 \mathrm{~kg} / \mathrm{s}$, the time-averaged velocity magnitudes at the main shroud entry are significantly increased as compared with the high mass-flow rate case, although less flow enters through the com- 


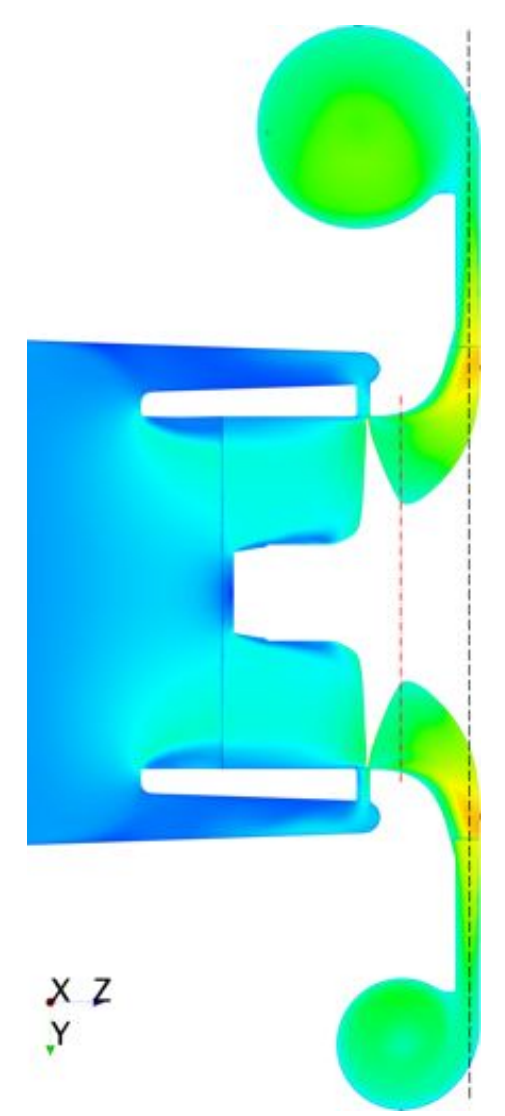

(a) $\dot{m}=0.27$

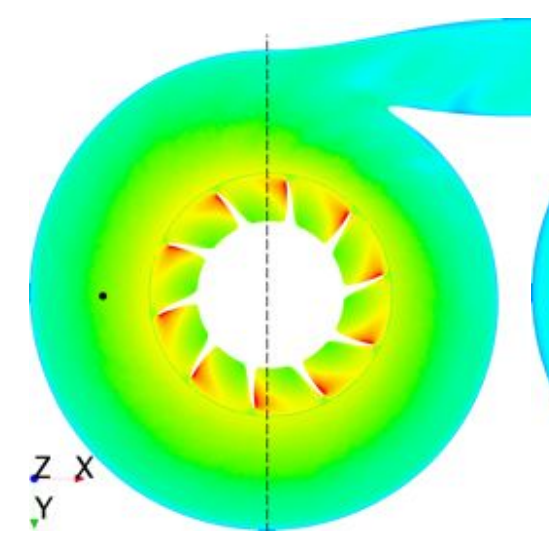

(d) $\dot{m}=0.27$

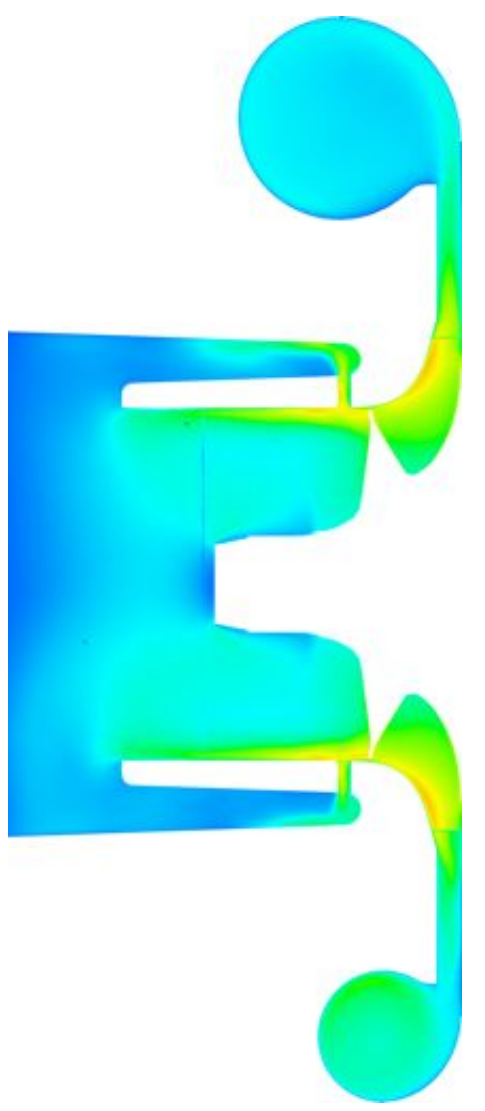

(b) $\dot{m}=0.105$

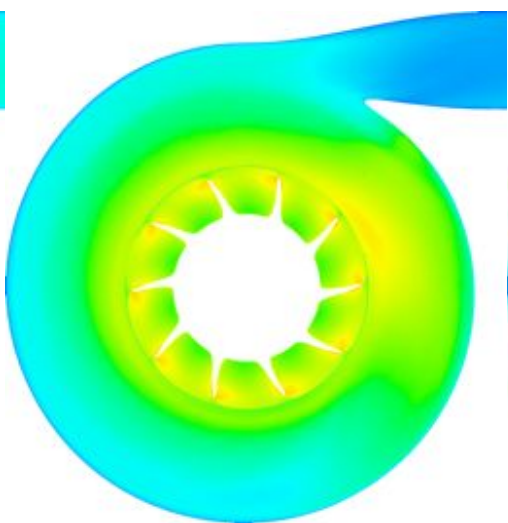

(e) $\dot{m}=0.105$

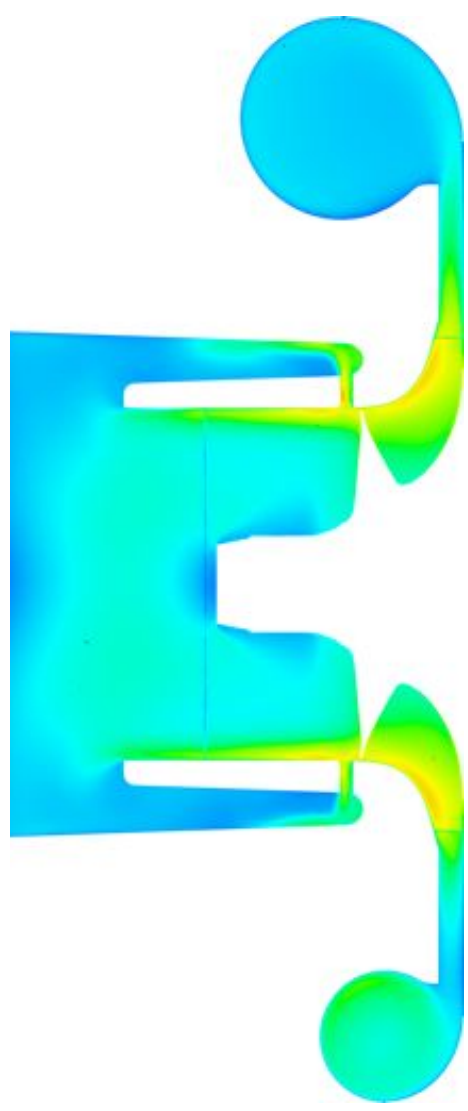

(c) $\dot{m}=0.05$

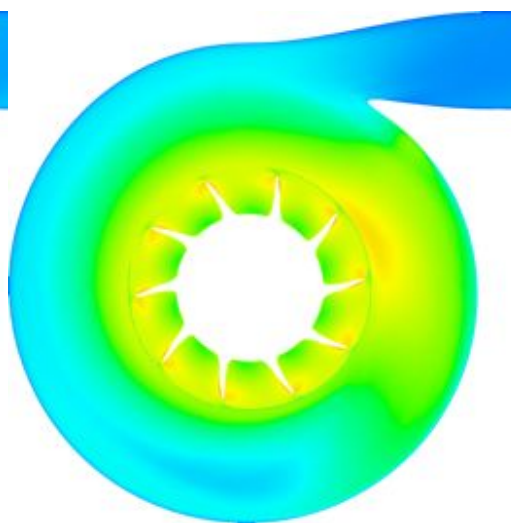

(f) $\dot{m}=0.05$
0.0000
66.000
time-averaged velocity magnitude $(\mathrm{m} / \mathrm{s})$
132.00
198.00
264.00
330.00

Figure 4: The time-averaged velocity magnitude $(\mathrm{m} / \mathrm{s})$ is show in the Figures (a) to (f) for different mass-flow rates at the speed line of $64 \mathrm{krpm}$. The locations of the cut planes with respect to each other are indicated by the dashed lines in the Figures (a) and (d). 


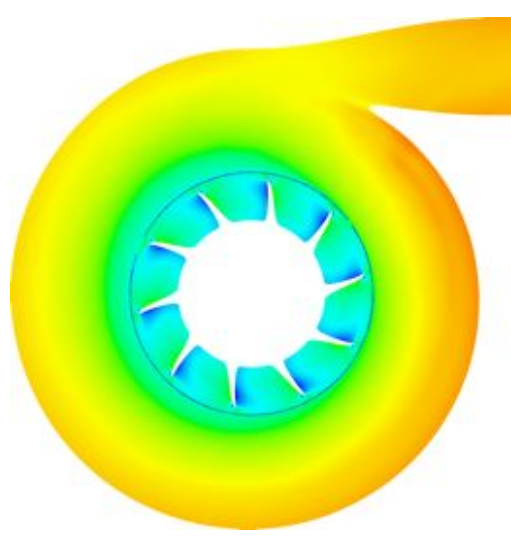

(a) $\dot{m}=0.27$

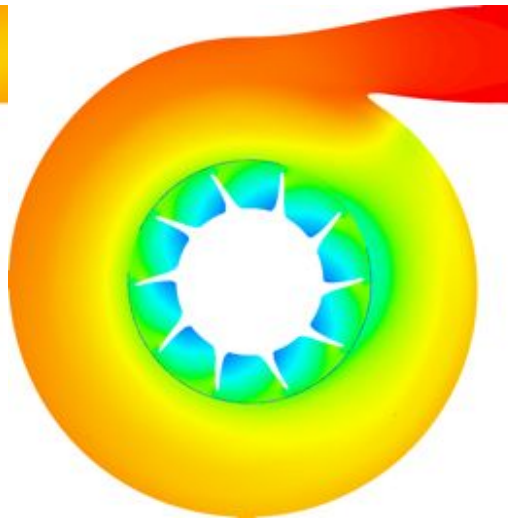

(b) $\dot{m}=0.105$

time-averaged static pressure $(\mathrm{Pa})$

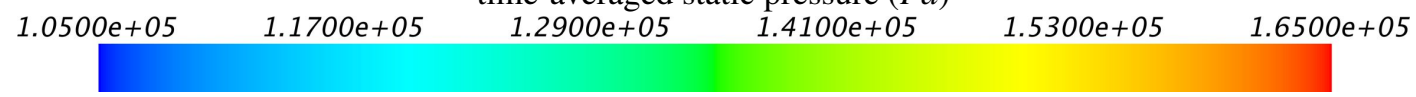

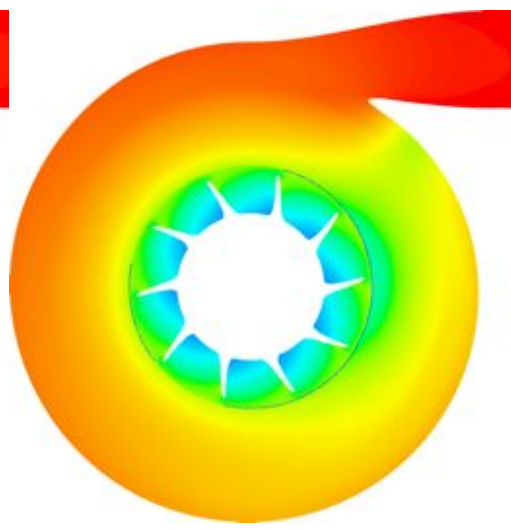

(c) $\dot{m}=0.05$ the Figures (a) to (c). The plane location is shown in Fig. 4.

pressor inlet. Even in the mid of the impeller, high velocity magnitudes can be observed in Fig. 4 (f), which occur due to confinement generated by the recirculating flow structures from the ported shroud. The high velocities at the outer periphery of the main shroud and impeller are increased compared to the case of $\dot{m}=0.105$ $\mathrm{kg} / \mathrm{s}$. A decreased amount of flow streaming from the diffuser into the volute can be observed especially towards the six o'clock location, where the flow velocities are remarkably lower in the diffuser outlet than in the volute. The cuts through the compressor diffuser shown in Fig. 4 (f) and Fig. 5 (c) depict that the high velocity region reaching out of the impeller at the two o'clock location is increased and the radial velocity gradients as well as the pressure gradients are increased. The velocity magnitudes at the outer periphery of the volute, starting at the six o'clock location towards the compressor outlet, are significantly decreased. A low velocity region at the bottom location of the volute can be observed.

For the high mass-flow rate case $(\dot{m}=0.27 \mathrm{~kg} / \mathrm{s})$, the instantaneous flow in the compressor inlet develops without any large-scale disturbances, as shown in Fig. 6 (a) and Fig. 6 (d). The flow detaches at the entrance of the main shroud and a recirculation region evolves. This flow restriction causes flow acceleration over the separation bubble. The extent of the separation bubble ends before the wheel blades are reached. Some flow is drained into the shroud cavity and recirculates to the compressor inlet. Unsteady flow motion can be observed in the shroud cavity. Nonetheless, the flow does not return in the mid plane towards the main shroud, moreover, as jet-lets leaving from the shroud rips, see Fig. 6 (a). The inlet flow is insignificantly disturbed by the recirculating flow for this operating condition. Noteworthy to observe is that no negative axial velocities occur in the impeller downstream of the bleeding slot. At the impeller mid, small flow disturbances are induced and drained into the blade passages. However, with the compression of the flow, the unsteady flow features are damped and rather laminar flow discharges into the diffuser. The flow streams without any notable disturbances into the volute. Remarkable is the formation of recirculation bubbles in the exit region of the impeller, which do not seem to have large influence on the flow further downstream. The occurrence of the flow reversal and the turbulence distribution in the impeller at design operating conditions has been also reported and analysed by Guleren et al. [26].

When the mass-flow rate is reduced to $\dot{m}=0.105$ $\mathrm{kg} / \mathrm{s}$, a distorted flow-field at the compressor inlet can be observed, as shown in Fig. 6 (e). The static separation bubble, which was previously identified at the impeller discharge under stable operating conditions, mass-flow rate of $\dot{m}=0.27 \mathrm{~kg} / \mathrm{s}$, cannot be seen any more, since negative axial velocities emerge from this location. The observed back-flow enters partially through the bleeding slot into the shroud cavity against the axial mean flow direction. A larger amount of fluid recirculates through the shroud cavity at amplified negative axial velocities as compared with the stable operating condition. The flow through the shroud cavity 


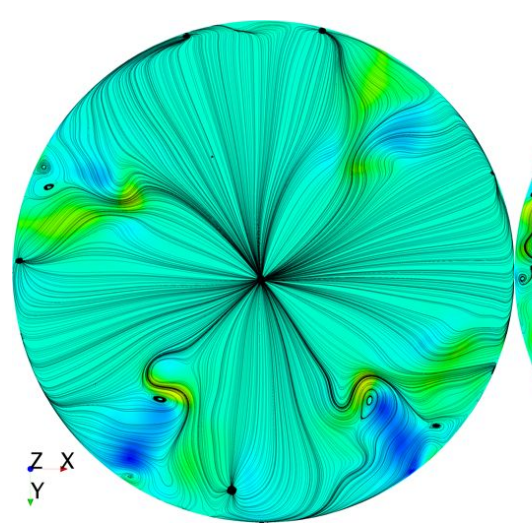

(a) $\dot{m}=0.27$

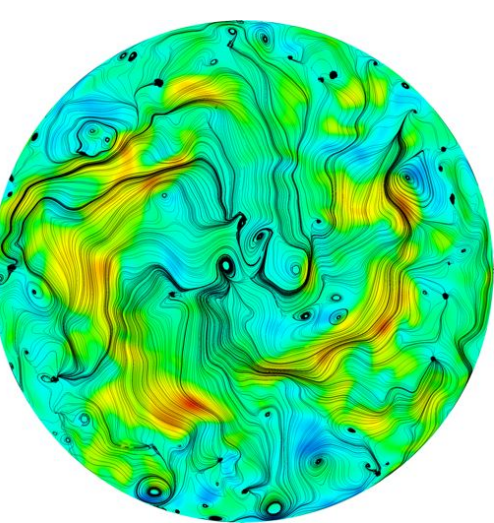

(b) $\dot{m}=0.105$

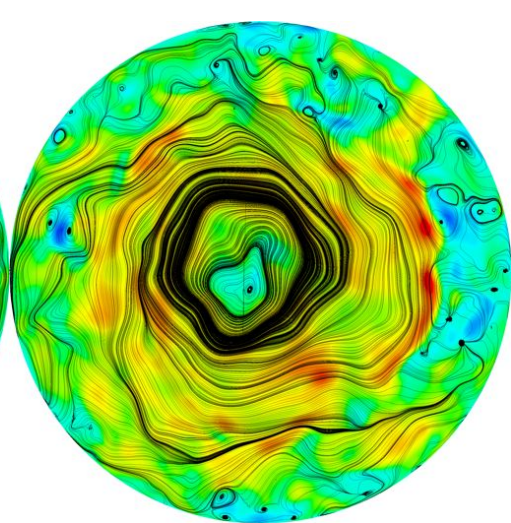

(c) $\dot{m}=0.05$
$-80.000$
$-32.000$
64.000
112.00
160.00

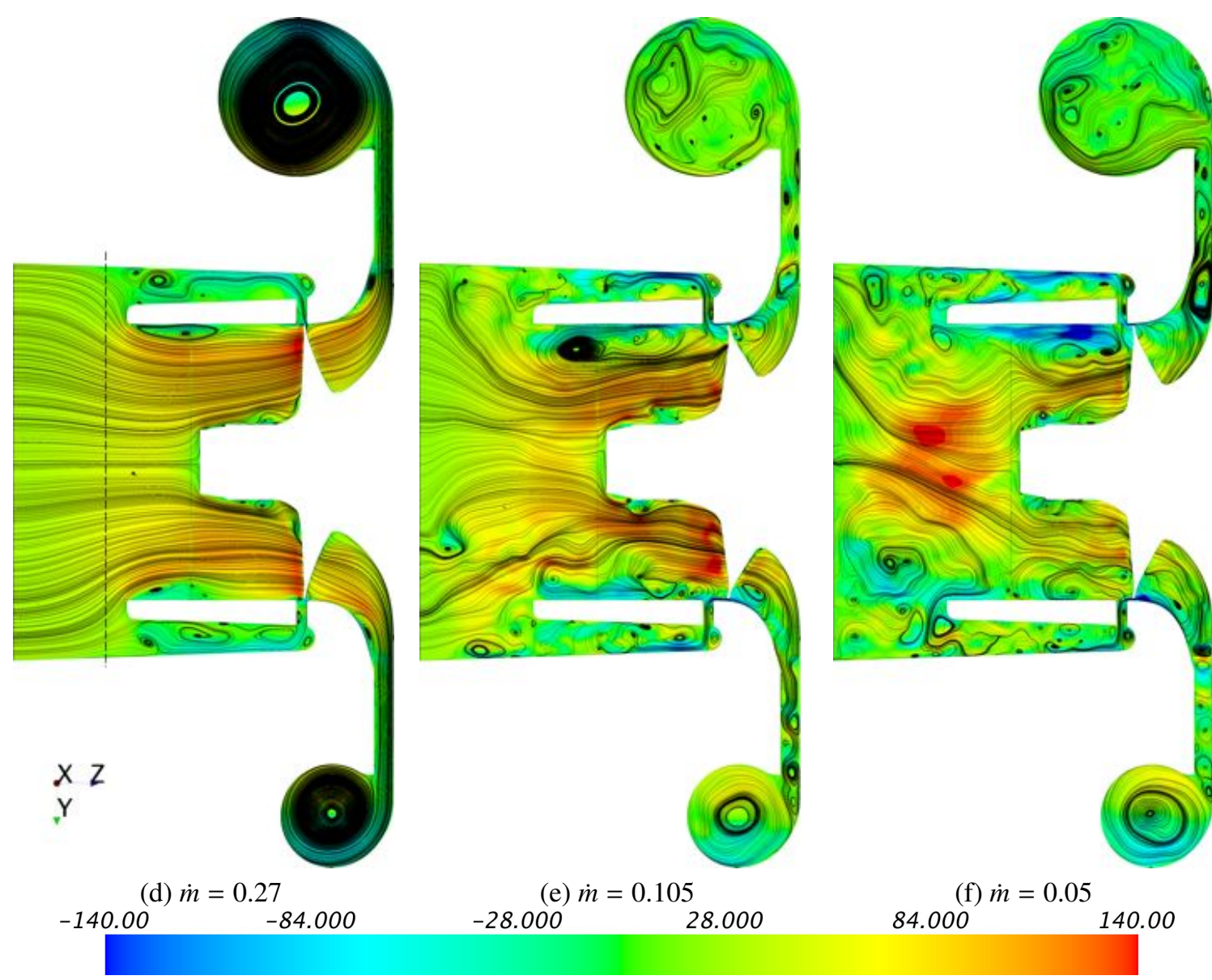

Figure 6: Characteristics of flow dynamics for stable and for low-mass-flow unstable operating conditions; Top row: streamlines on top of the tangential velocity $(\mathrm{m} / \mathrm{s})$ in cut plane (the location is indicated by a dashed line in Fig. (f) ). Bottom row: streamlines on top of the instantaneous axial velocity $(\mathrm{m} / \mathrm{s})$ component are shown in a vertical mid-plane view (same plane as shown in Fig. 4 (a) to (c)). 
interacts and disturbs the flow-field at the compressor inlet. Further, negative axial velocities can be observed at the periphery of the main shroud, which cause large recirculation zones at the main shroud entry. Naturally, the axial back-flow returns from the impeller passages towards the inlet with a tangential velocity component. This causes a global rotating flow-field at the compressor inlet, which is shown in Fig. 6 (b). Hence, the flowfield develops non-uniform towards the blades. The unsteady flow features develop mainly at the outer radii in the blade passages, whereas the flow towards the centreline is less effected by the disturbances. The flow reaching the diffuser is highly disturbed, as illustrated in Fig. 6 (e). Unsteady flow features are noticeable, which propagate through the diffuser into the volute.

Figure 6 (f) shows that unsteady flow motion can be observed in the entire region of the compressor inlet for the lowest mass-flow rate simulated $(\dot{m}=0.05 \mathrm{~kg} / \mathrm{s})$. At the bottom, vortical flow structures of the size of the blade passage are blocking the inflow and also at the top large structures are influencing the inflow. Therefore, high axial flow velocities in the mid of the compressor inlet are induced, which result in enhanced unsteady flow structures evolving from the mid of the impeller. A large amount of flow recirculates through the shroud cavity and high back-flow velocities $(-140 \mathrm{~m} / \mathrm{s})$ can be observed in Fig. 6 (f). Also in this case, a large proportion of the mass-flow entering into the shroud cavity originates from far downstream of the impeller section streaming against the main flow direction along the shroud into the bleeding slot. Further, some flow streaming against the main axial streaming direction can be observed in the main shroud. Figure 6 (c) shows the strong swirling flow motion at the compressor inlet induced by the reversed flow. The diffuser section is dominated by unsteady flow motion. At the bottom of the Fig. 6 (f), the streamlines indicate that no flow enters from the diffuser into the volute at this time instant and at the top, the flow streams into the volute at a sharp angle, against the expected swirling direction. The flow entering into the volute at the top has a lower momentum and exhibits a flapping behaviour, where the stream flows at times against and at other times, in the expected swirling direction in the volute. It is noteworthy to mention that the undisturbed swirl in the bottom part of the volute occurs at most times, whereas the flow in the upper section of the volute is mostly disturbed.

Instantaneous static pressure contours are shown in Fig. 7 in a cut view through the impeller. For the high mass-flow rate operating condition $(\dot{m}=0.27 \mathrm{~kg} / \mathrm{s})$ shown in Fig. 7 (a), a similar static pressure distribution can be observed for each blade passage. In front of the pushing blade, a high-pressure region results, especially towards the blade tips. At the suction side of the blade tips, a local low-pressure region is induced. The inplane streamlines on top of the pressure contours do not indicate large vortical structures for this stable operating condition. In this cut plane, the static pressure distribution changes significantly when the mass-flow rate is reduced to $\dot{m}=0.105 \mathrm{~kg} / \mathrm{s}$. A higher static pressure zone establishes (preceding the blades) at the outer periphery of the impeller. However, this high-pressure zone does not reach until the blade geometry. Behind the blades, the static pressure is lowered, but remains higher than in the blade passage region by the hub, where the lowest static pressures occur. The lowest static pressures in the blade passage are induced at the three o'clock location. These low-pressure cells are propagating circumferentially and rotate with the frequency of $250 \mathrm{~Hz}$. The time-averaged pressure data presented in Figs. 5 $(b, c)$ are calculated for each individual computational cell and the impeller mesh is rotating. This explains the depicted time-averaged static pressure contours. A lower static pressure zone outside of the wheel at three o'clock can be observed in Figs. 5 (b, c), which implies that a lower static pressure dominates in the wheel passage at about three o'clock position. A significant amount of vortical flow generation can be seen at the blade tips by inspecting the streamlines shown in Fig. 7 (b). Similar features of the static pressure distribution can be observed in Fig. 7 (c) when the mass-flow rate is reduced to $\dot{m}=0.05 \mathrm{~kg} / \mathrm{s}$ as for the operating condition $\dot{m}=0.105 \mathrm{~kg} / \mathrm{s}$. The occurring static pressure at the outer periphery is higher than for the previously inspected operation condition. This correlates with the higher pressure region developed in the region between six and one o'clock of the volute and diffuser for this operating condition.

At high mass-flow rates $(\dot{m}=0.27 \mathrm{~kg} / \mathrm{s})$, the flow streams smoothly from the diffuser into the volute throughout the entire circumference, as shown by streamlines plotted in the diffuser and volute in Fig. 8 (a). The flow leaving the wheel exhibits a high radial velocity component, which contains enough kinetic energy to overcome the adverse pressure gradient. Therefore, an undisturbed large swirling motion is generated in the volute, which is conserved until the outlet of the computational domain. The swirling motion is initiated at the tongue and no recirculating flow back into the volute over the tongue can be observed for this operating condition. As shown in Fig. 8 (b) for the lowest massflow rate simulated ( $\dot{m}=0.05 \mathrm{~kg} / \mathrm{s}$ ), the adverse pressure gradient is significantly increased and therefore, the radial velocity component is drastically reduced. The 


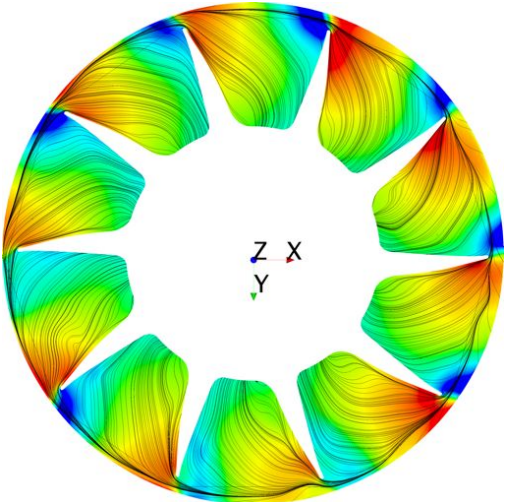

(a) $\dot{m}=0.27$
95000 .
$1.0100 e+05$

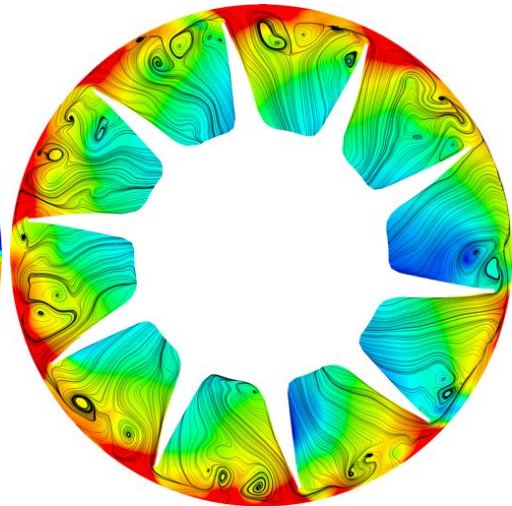

(b) $\dot{m}=0.105$

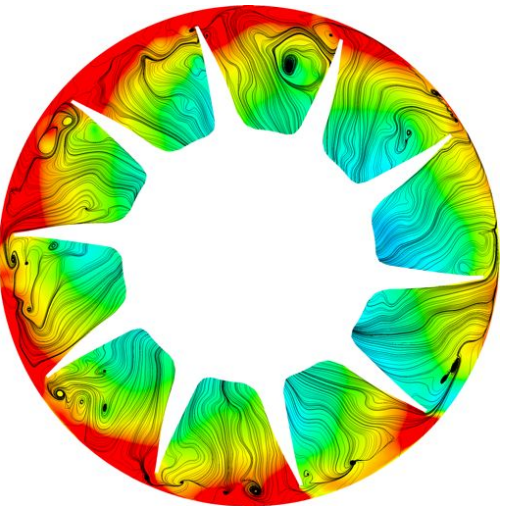

(c) $\dot{m}=0.05$ $1.2500 e+05$

Figure 7: The streamlines on top of the instantaneous static pressure $(\mathrm{Pa})$ are shown in a cut view through the impeller. The location of the cut plane is indicated in Fig. 4 (a) by the dash-dotted line.

highest radial flow velocities for this operating condition can be noted between three and four o'clock close to the impeller. However, it can be observed that the streamlines exhibit a large tangential component. Further, the streamlines leaving the diffuser into the volute at the three o'clock location, entered into the diffuser in the region between nine and twelve o'clock. Hence, the flow travels a longer distance before discharging into the volute for this operating condition. In regions where the flow velocities are low, unsteady flow evolves in the diffuser. A large amount of recirculating flow can be noted from the volute end around the tongue region, where the highest negative radial velocities occur. Further, the swirling motion in the volute is lower for this operating condition compared to the high mass-flow rate condition, since the flow enters into the volute with an enhanced tangential component and with a reduced radial velocity component.

The power spectral density of the velocity signal sampled in the probe located at the nine o'clock location in the diffuser is shown in Fig. 9 for the three operating conditions. All the spectra exhibit the blade passing frequency at $10.666 \mathrm{kHz}$ and the first harmonic. Except these peaks no other dominant peaks are visible for the high mass-flow rate case. The velocity fluctuations in the low frequency range increase significantly for the lower mass-flow rate cases. High velocity fluctuation amplitudes can be observed in the neighbourhood of $500 \mathrm{~Hz}$ for the case $\dot{m}=0.105 \mathrm{~kg} / \mathrm{s}$ and around 600 $\mathrm{Hz}$ for the case $\dot{m}=0.05 \mathrm{~kg} / \mathrm{s}$.

Two flow decomposition methods, i.e. POD and DMD, are utilised to extract the coherent flow features
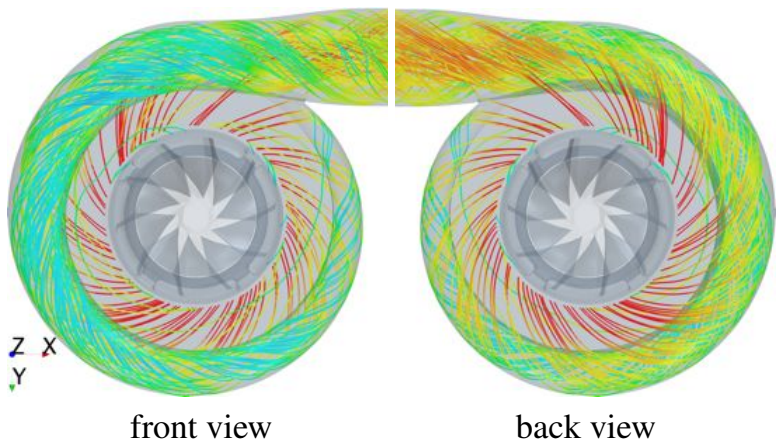

(a) $\dot{m}=0.27$

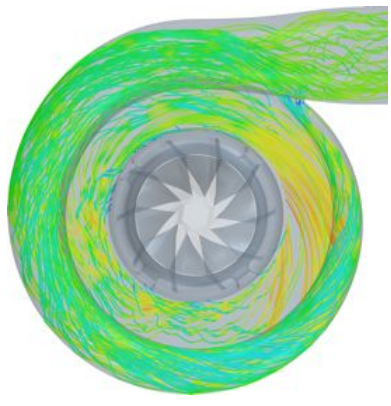

front view

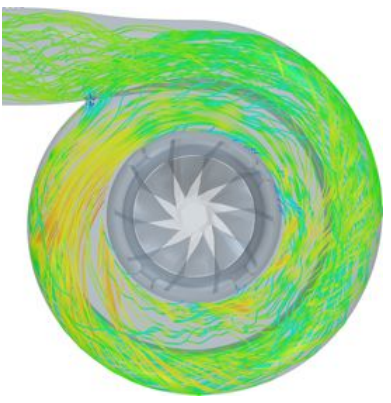

back view (b) $\dot{m}=0.05$

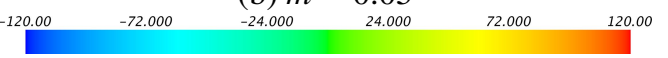

Figure 8: Streamlines in the diffuser and the volute region coloured by the radial velocity $(\mathrm{m} / \mathrm{s}$ ) for stable operating conditions (a) and unstable operating conditions (b).

responsible for the magnitude amplification shown in Fig. 9. The POD decomposition is the discretised finite 


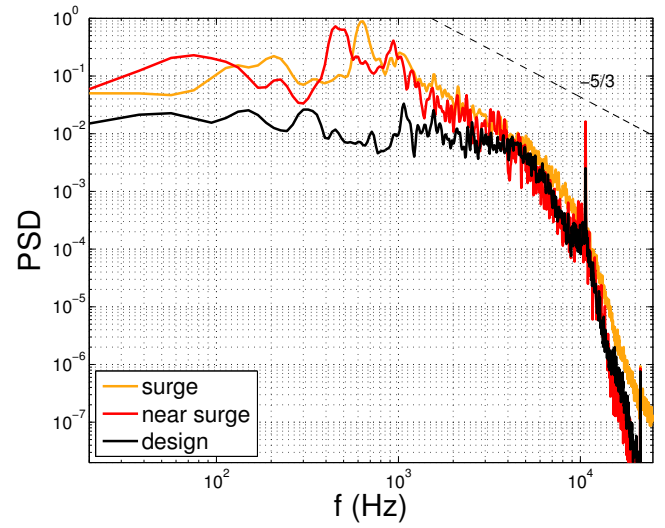

Figure 9: The power spectral density of the monitored velocity signals for the three operation conditions are shown. The probe location is indicated in Fig. 4 by a black dot.

interpretation of the Karhunen-Loéve theorem, which optimally defines a series of orthogonal functions with random coefficients to describe a stochastic process. Hence, the flow-field in terms of instantaneous snapshots is decomposed into a sum of spatial orthogonal modes (topo modes) and time coefficients (chrono modes). Hence, the spatial and temporal information of unsteady flow is separated and therefore, the coherent information of the spatial modes and the time dependency can be analysed individually. A drawback of the POD method is that the technique cannot identify structures responsible for phenomena occurring solely at one particular frequency. However, the DMD method provides the modes associated with unique frequencies and therefore supplements the POD analysis. Further, Schmid [27] showed that the DMD method retains its capability of extracting the most significant mode shapes even on reduced spatial domains.

The main focus of the mode decomposition analysis was held on the lowest mass-flow rate case $\dot{m}=0.05$ $\mathrm{kg} / \mathrm{s}$. The low frequency range of the spectra is of interest when investigating the surge phenomenon. Hence, the snapshot samples used were gathered at consecutive time steps of $0.234 \mathrm{~ms}$ for 1025 instants in order to capture a significant proportion of the low frequency range. The highest frequency captured is approximately $2 \mathrm{kHz}$ and the lowest frequency contained in the analysis is approximately $4 \mathrm{~Hz}$. Hence, only low frequency phenomena are expected to be captured in the modal decomposition analysis. Sundström et al. [18] showed by a comparison with experimental data that this frequency range was reliably captured with the employed mesh resolution.

The modal flow decomposition analysis in the volute has been performed also for the highest mass-flow rate case simulated $\dot{m}=0.27 \mathrm{~kg} / \mathrm{s}$ with the same setup as previously described. However, no large-scale coherent structures in the diffuser (between impeller and volute) or the volute have been observed. The temporal mode amplitudes are spread over a wide range, without exhibiting significant spectral information. The largest flow structures are detected in the conical diffuser downstream of the volute. Therefore, only the results for the lowest mass-flow rate case $\dot{m}=0.05 \mathrm{~kg} / \mathrm{s}$ are presented.

The zeroth POD mode representing the ensemble averaged flow-field contains about 90.8 percent of the total flow energy and the shape is depicted in Fig. 10 (a) in terms of the tangential and radial components, where the streamlines of the POD mode indicate the directionality of the mode. The decomposition into these two components reveals the occurrence of low flow velocities towards the compressor outlet. The lowest negative radial component of the mode occurs under the tongue region, where the large static pressure gradient was observed. The highest radial component occurs at the three o'clock location. Worthwhile to note is that the streamlines shown for the zeroth POD mode indicate a division of the mass-flow just upstream of the tongue region with part of the flow going towards outlet and part of it being recirculated in the diffuser and the volute.

Since the POD modes represent the total velocity field in terms of a sum, the zeroth POD mode can be seen as base flow, where the higher modes represent deviations superimposed on the mean flow-field. The amplitude of the higher modes is illustrated in Fig. 11 (b) quantifying the importance of the modes with respect to each other in terms of a ranking accordingly to their amplitude. The temporal mode component states the variation of the mode shape in time. The power spectral density from the temporal POD modes revealing the dominant frequency dependence is shown in Fig. 11 (a) for the low mass-flow rate case of $\dot{m}=0.05 \mathrm{~kg} / \mathrm{s}$. Colour code indicates the leading ten modes. The highest peak is represented by the first two POD modes and occurs approximately at $600 \mathrm{~Hz}$. Several peaks at a frequency of about $1 \mathrm{kHz}$ are represented by the third and fourth POD modes. The fifth POD mode is the first low frequency mode and is associated with a wide range of frequencies between 100 and $400 \mathrm{~Hz}$. Low frequency peaks in the range of $40 \mathrm{~Hz}$ to about $100 \mathrm{~Hz}$ can be observed, which are represented by the ninth POD mode.

With Figure 11 (a) it is verified that the first two POD mode coefficients have the same spectral properties. The plotted chrono modes of the first two POD modes exhibit similar properties in time and only a small phase shift between the chrono modes can be perceived. Fur- 

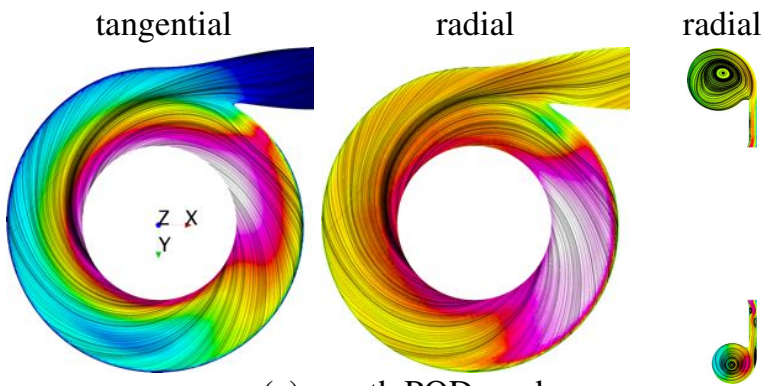

(a) zeroth POD mode
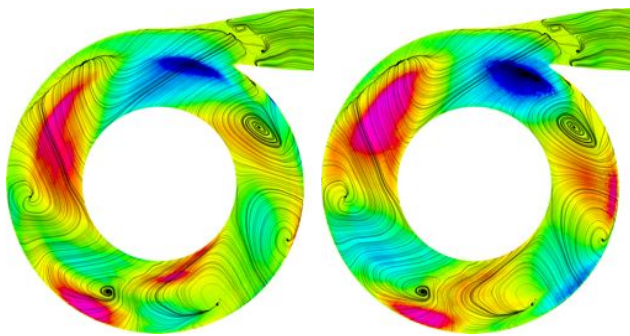

(b) $1^{s t}$ POD mode
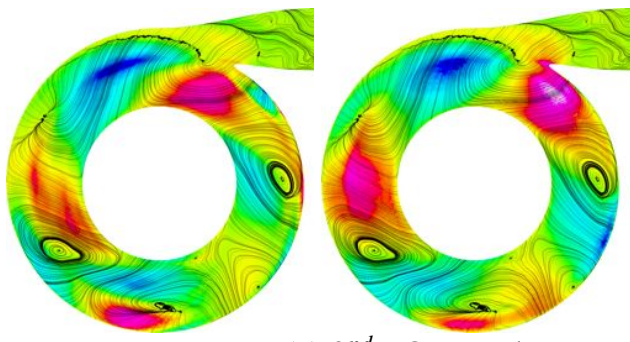

(c) $2^{\text {nd }}$ POD mode
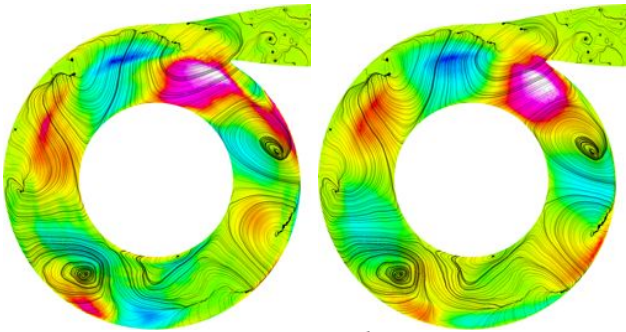

(d) $3^{\text {rd }}$ POD mode
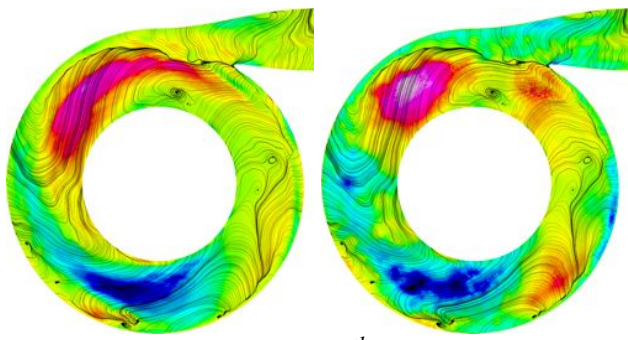

(e) $9^{\text {th }}$ POD mode

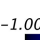

$-0.60$

$-0.20$

0.20

0.60
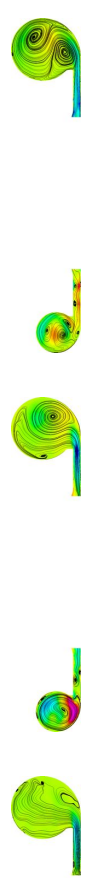

Figure 11: The power spectral density of the time coefficients result-

Figure 10: The shapes of selected POD modes is illustrated for $\dot{m}=$ $0.05 \mathrm{~kg} / \mathrm{s}$, by showing the tangential and the radial component of the modes. Only the tangential mode of the zeroth mode is shown from zero to the maximum value. All the other mode shapes are normalised. ing from the POD analysis for the lowest mass-flow rate case $\dot{m}=0.05$ $\mathrm{kg} / \mathrm{s}$ is shown in Fig. a. The leading ten modes are indicated in colour, while the higher modes are plotted in black. The kinetic flow energy content of the first twenty modes is shown in Fig. (b).

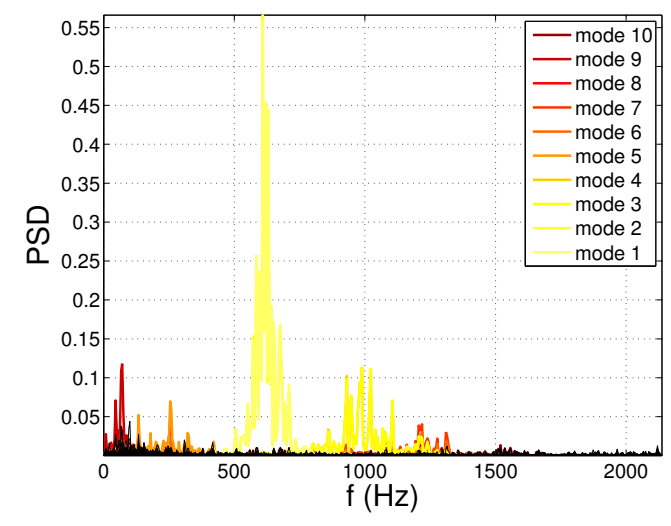

(a) POD mode spectra

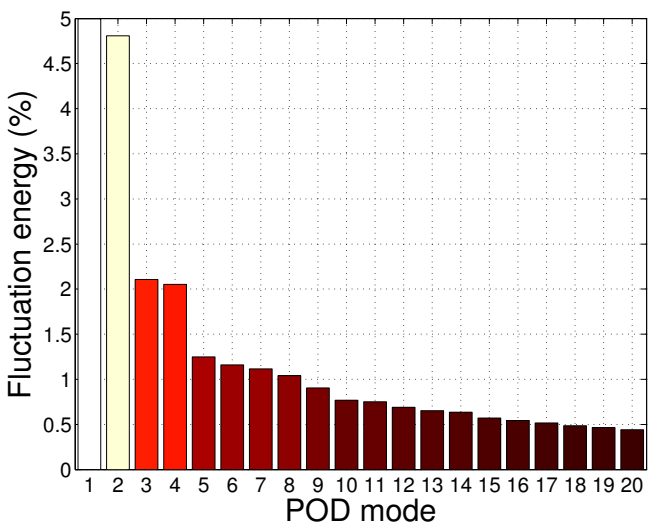

(b) POD mode energy

ther, the time coefficients of the first and second POD mode, shown in Fig. 12, exhibit that the chrono modes oscillate over the average value, zero. The shapes of the leading first and second POD modes are shown in Fig. 10 (b) and (c), respectively. The highest magnitudes of the topo modes are located in the transition between the diffuser and the volute. Since the time coefficient varies sign, the streaming direction induced by the modes changes. A star-like shape with three point can be noted with three regions of positive and three of negative values, respectively. There is a slightly shift between the first and the second POD mode shapes in the streamwise direction. The second POD mode always precedes the first POD mode as indicated by the chrono modes in Fig. 12. The modes can be interpreted as leaking and repulsing flow shapes, when superimposed onto the zeroth mode. 


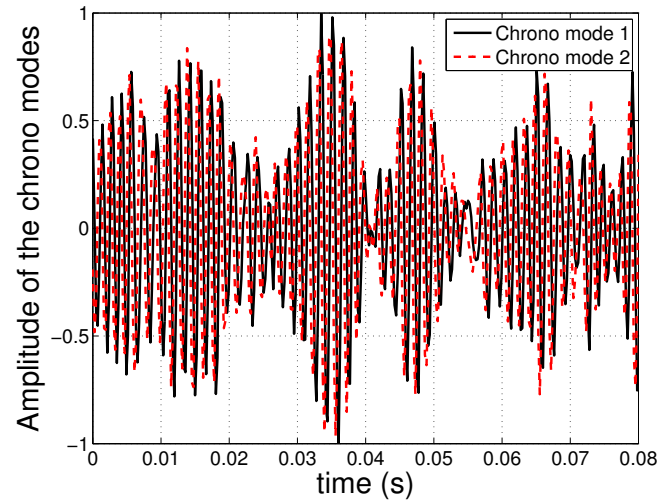

Figure 12: The phase shift of the time coefficients for the leading two POD modes is indicated by plotting the time coefficients in a restricted time interval.

The third and fourth POD modes represent the same flow mechanism at a higher frequency of about $1 \mathrm{kHz}$. The chrono modes are phase shifted against each other and so are the topo modes, as analogously shown for the first and second POD modes. The shape of the third POD mode is show in Fig. 10 (d). Once more, the highest magnitudes are accumulated in the transition between diffuser and volute and the magnitudes form with the streamlines a star with four points. Higher POD mode pairs are observed at higher frequencies, where the star shape increases the number of points with increasing frequency.

The POD mode associated with the low frequency range in between $40 \mathrm{~Hz}$ to $100 \mathrm{~Hz}$ is depicted in Fig. 10 (e) and contains the $9^{\text {th }}$ highest energy content of the fluctuating flow energy (see also Fig. 11 (b)). The shape of this POD consists basically of two large structures, one in the upper half and another in the lower half of the compressor domain. The tangential and radial components for each of the structures are opposing each other. Hence, while one structure represents an increased stream out of the diffuser into the volute, the other structure indicates a reduced flow out of the diffuser into the volute on top of the zeroth POD mode.

The radial mode components in the vertical cut illustrate the flapping of the flow discharging from the diffuser into the volute. The mean flow, represented by the zeroth POD mode, exhibits that the flow streams from the diffuser into the volute without detaching from the outer volute walls. For the leading POD modes, the swirling stream in the volute is disturbed and the flow separates from the outer volute walls.

The spectra of the DMD analysis, shown in Fig. 13 (a), exhibits the magnitudes associated with the indi- vidual modes corresponding to discrete frequencies. A similar amplitude distribution as for the POD decomposition and the velocity point spectra results, verifying the modal analysis outcome. The highest amplitudes captured are associated with frequencies around $600 \mathrm{~Hz}$. The peak visible in the DMD spectra at about $2133.2 \mathrm{~Hz}$ (furthest to the right), results due to aliasing of the blade passing frequency to a lower harmonic and is therefore not physical. Also low frequency peaks around $130 \mathrm{~Hz}$ are visible, as well as peaks around $1 \mathrm{kHz}$.

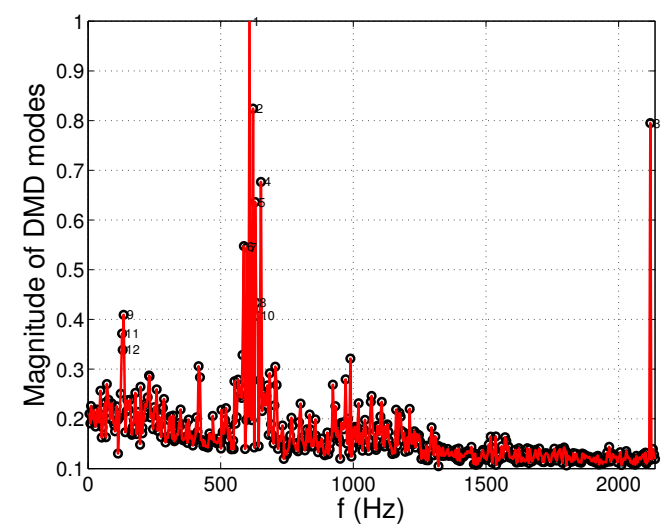

(a) DMD mode spectra

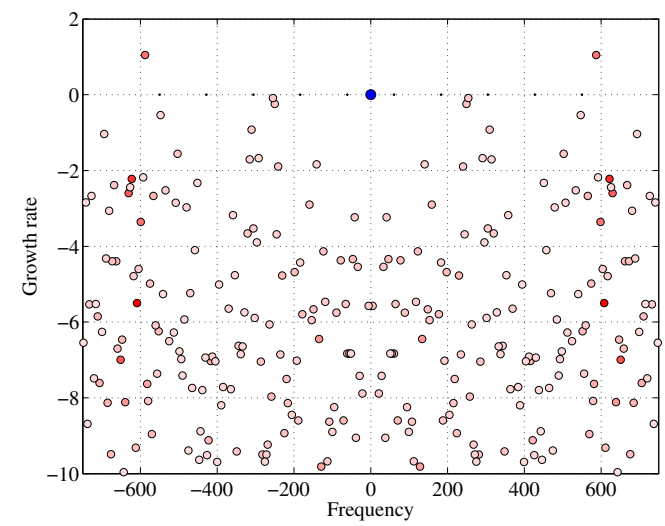

(b) DMD mode growth rate

Figure 13: The figures refer to the lowest mass-flow rate case $\dot{m}=$ $0.05 \mathrm{~kg} / \mathrm{s}$. The mode magnitudes and the growth rate of the DMD modes are shown in Fig. (a) and Fig. (b), respectively.

The leading two DMD modes expose the same flow phenomena as the leading two POD modes, since the shape of the modes is similar. However, the order of the modes is reversed and the amplitudes are slightly changed. The frequency peak at about $130 \mathrm{~Hz}$ is represented by three DMD modes, i.e. the $9^{\text {th }}$, the $11^{\text {th }}$ and the $12^{\text {th }}$ DMD mode, which have a similar shape and therefore only the $9^{\text {th }}$ DMD mode is shown in Fig. 14 
(c). The tangential mode component shape exhibits high magnitudes at the passage from the diffuser into the volute in streamwise direction from the six o'clock location up to the one o'clock location. Further, the tangential mode component has opposed values in the sector region from the two o'clock location to the four o'clock location where high velocity magnitudes have been observed in the averaged flow-field, i.e. the zeroth mode. Also under the tongue, negative tangential components can be observed. The global distribution of the radial mode component is similar to the tangential component. The highest magnitudes of the mode occur in the near region of the tongue, between diffuser and volute. The higher modes are a harmonic oscillatory superposition of the zeroth mode, which represents the mean flowfield. Hence, the sign of the mode shape changes. Thus, this mode is thought of a surge mode, where the volute and the diffuser is emptying and refilling at this frequency.

The growth rate of the DMD modes can be associated with the influence of the mode triggering flow instabilities. Most of the computed DMD modes in this analysis exhibit a negative growth rate, which is shown in Fig. 13 (b). Hence, the modes are stable in time and the amplitudes mode will decay. However, the growth rate of the $6^{\text {th }}$ DMD mode at a frequency of $600 \mathrm{~Hz}$ is positive. Hence, the flow instabilities induced at this frequency will evolve amplified with progressing time.

\section{Discussion}

LES simulations have been performed with the aim to identify flow features leading to surge. Consecutive comparison of the flow-field revealed distinctive flow features leading to surge. The observed flow features have been separately described in detail in the previous section. In this section, the individual flow features are linked together forming a global flow phenomenon, i.e. surge. The most important flow features found with decreasing the mass-flow rate are the increasing radial pressure gradient in the diffuser and the imposed rotation of the entrained flow into the impeller by the backflow.

At operating conditions near surge, the mass-flow rate through the diffuser remains constant and the radial pressure gradient manifesting in the diffuser is static. However, the mass-flow rate starts oscillating when approaching surge and the radial pressure gradient in the diffuser exhibits oscillations as well revealing gas compressibility properties. In the present investigation, modal flow decomposition methods have been used to

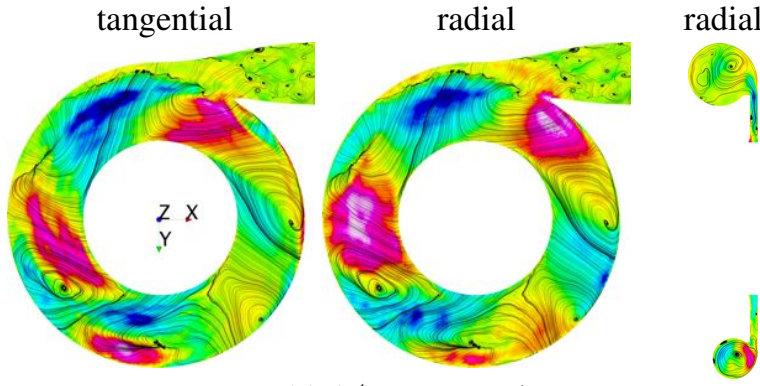

(a) $1^{\text {st }}$ DMD mode
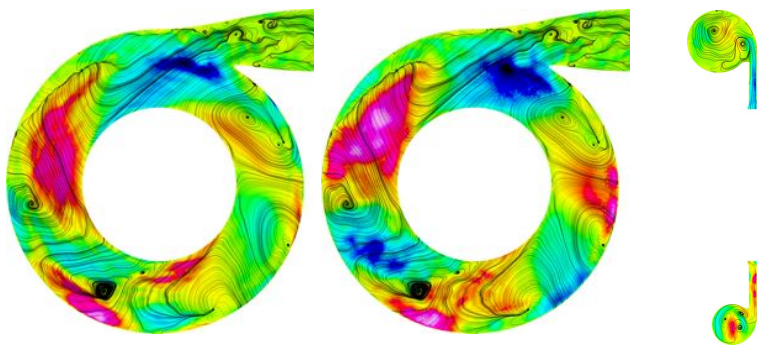

(b) $2^{\text {nd }} \mathrm{DMD}$ mode
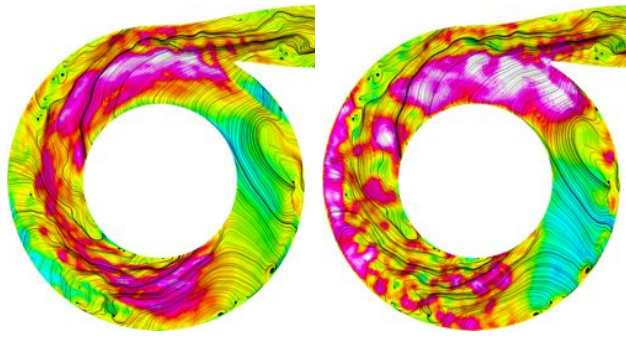

(c) $9^{\text {th }}$ DMD mode

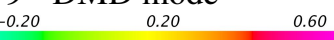
1.00

Figure 14: The shapes of the DMD modes is shown in the same manner as Fig. 10.

describe the unsteady flow features at off-design operating conditions in a centrifugal compressor. The dynamic mode decomposition exhibits modes corresponding to the surge frequency, where the shape of the modes illustrate the filling and emptying of the flow. The static pressure in the volute fluctuates in a wave-like motion periodically. It increases and decreases successively at regular time intervals starting from the compressor outlet region and continuing into the volute in the counter clockwise direction, before the cycle repeats. The frequency of the observed pattern is the same as the surge frequency. At times with the high static pressure present in the volute section, the flow streaming from the diffuser into the volute is decreased.

A high radial pressure gradient in the radial diffuser leads to reduced flow momentum at low mass-flow rates. If the flow pushed downstream by the impeller does not have enough flow momentum to overcome the 
pressure gradient in the diffuser, the flow direction is reversed at the impeller discharge and a tip leakage over the compressor blades establishes. The occurrence of such a flow reversal during off-design operating conditions at the outer periphery of the impeller is in agreement with the observations by Despres et al. [10] using numerical flow simulations to investigate a different geometry. The tip leakage recirculations are increased towards lower mass-flow rates and lead to a distorted swirling flow at the inlet of the compressor, which result in a reduced performance of the impeller. The induced swirling motion in the centrifugal compressor inlet has been shown experimentally by Gancedo et al. [17] using PIV measurements. A swirl upstream of the impeller affects the blade efficiency by changing the incident angle of the flow. Galindo et al. [7] observed extended surge margins when the inlet flow is swirled against the rotation direction of the wheel, while the swirl did not improve the compressor efficiency at design conditions. The presented data explains that counteracting the swirling motion provoked at off-design conditions extends the surge line margins of a centrifugal compressor by improving the operation potential of the impeller. Commonly, a bypassing channel recirculating flow from the end of the compressor to its inlet is used to avoid deep surge. The present data suggests that the bypassed stream is fed back in a manner that a counteracting swirl is induced improving the efficiency of the impeller blades.

Based on the observations made with this investigation, the surge phenomenon can be described as follows: within a surge cycle, high pressure rises in a standing sinusoidal waveform from the outlet and builds up towards the compressor. This corresponds to the filling of the compressor. The high pressure build up continues progressing in the volute against the rotation direction of the wheel, i.e. from the exit pipe following the annular shape of the volute towards the tongue. However, it was shown that the presence of the high-pressure region at the volute exit generates a high-pressure gradient area with the lower pressure under the tongue towards the initial volute geometry. This forces the flow to stream under the tongue back into the volute and a separation bubble at the outer periphery forms. The establishment of the separation bubble is therefore related to the surge frequency and enhanced activity related to the surge frequency can be found in this region. There, the recirculating stream accelerates in the direction of the wheel rotation. Hence, this generated stream pushes against the arriving pressure rise propagating in the opposite direction of the wheel rotation in the volute. Since the flow is held back at the interface between dif- fuser and volute by the increased pressure building up from the outlet, the flow can only effectively discharge into the volute in the region between three o'clock and five o'clock. Therefore, the high velocity region is captured in this sector for the off-design conditions. The DMD analysis performed by the authors describes this phenomenon and the corresponding mode was exposed. With the pressure increasing in the volute also the pressure rises in the radial diffuser towards the impeller. It was shown that at the outer periphery of the impeller, the increased pressure pushes the flow against the main streaming direction towards the compressor inlet. Thus, a high speed leakage following the shroud walls streams out against the main flow direction towards the inlet of the compressor. Naturally, it swirls with the impeller rotation. Part of this near-wall back-flow is recirculated through the ported shroud cavities back into the main flow. Nevertheless, the strong swirling motion of the leakage flow enhances the swirling motion of the incoming flow upstream of the impeller, which will swirl in the same direction as the impeller rotates. Thus, the flow incidence angles are altered and the effectivity of the wheel to push fluid downstream is reduced. The reduced amount of fluid being delivered downstream causes a pressure decrease in the diffuser, volute, and exit pipe system, which corresponds to the emptying of the compressor. With the reduction of the pressure in the downstream system, the amount of fluid pushed as tip leakage upstream is also decreased. Hence, the swirl upstream of the impeller is weakened and the efficiency of the wheel blades to deliver fluid downstream increases. To our understanding, this cyclic sequence of events manifests globally in form of a limit cycle as surge.

The understanding of the surge phenomenon is important. However, in order to prevent the occurrence of surge, the precursors need to be analysed carefully. Worthwhile to note is that the time-averaged velocity and pressure fields did not change drastically in between the operating condition close to and at surge. Along a speed line the impeller rotates with the same speed and hence, similar amounts of fluid are expected to be pushed downstream. Both operating conditions, close to and at surge, revealed back-flow in form of tip leakage, which are provoked by the high pressure gradient. However, the swirling flow oscillations in the impeller eye and standing pressure waves in the exit pipe are significantly amplified at the surge operating condition, while a global oscillation at a distinct frequency is not observed at the near surge operation condition. This implies that the overfilling process in form a pressure increase in the exit pipe does not occur at operating con- 
ditions prior to surge, since the mass-flow oscillations are allowed to leave through the outlet boundary before damming up to large amplitudes. This flow restriction by the outlet boundary initiates an enhanced pressure gradient at surge, which results in enhanced back-flow and the alteration of the blade incidence angles, which result in a harmonic limit cycle oscillation.

For the off-design operating conditions, the monitored velocity history in a probe location in the diffuser revealed a significant peak at approximately the frequency corresponding to half of the rotating order. Raitor and Neise [28] investigated the narrowbanded tip clearance noise, which was reported to manifest at off-design operating conditions in this frequency range. The modal analysis revealed associated modes dominant at this frequency range corresponding to a leaking mechanism taking place between the diffuser and the volute at these frequencies. Near surge, the flow momentum starts to be too low to discharge at all times from the diffuser into the volute. Therefore, the flow is redirected to the sides and leaks only at certain times into the volute. This unsteady flow motion is described by the extracted modes corresponding to the leaking mechanism between diffuser and volute. Using modal analysis, the frequency of these events could be determined. At deep surge, the global oscillating motion of the pressure distribution become dominate and this local phenomenon of flow leaking form the diffuser into the volute at higher frequencies than the surge frequency is expected to be less prominent.

\section{Conclusions}

Large eddy simulations analysing the compressible flow-field development in a centrifugal turbocharger compressor approaching surge at a constant speed line have been presented. The entire compressor geometry has been included in the simulation process, where the sliding mesh technique has been employed to handle the rotation of the wheel. The outlet pipe attached to the compressor was dimensioned rather short (as compared with previous experimental investigations of the same compressor) and therefore, a mild surge is provoked at a relatively high frequency. The flow-field obtained at stable higher mass-flow rates is compared to operating conditions close to and at surge. The alterations of the flow-field approaching the low mass-flow range can give indications for the triggering mechanism leading to surge. Modal decomposition techniques have been used to investigate the unsteady flow phenomena in more detail. The main observations can be summarised in the following main points:
- Higher radial static pressure gradients establish in the diffuser at the off-design operating conditions, which counteract the flow momentum delivered downstream by the impeller.

- Similar time-averaged flow-fields (in terms of velocity and pressure) are observed close to and at surge. Back-flow in form of tip clearance leakage over the impeller blades is induced by the high radial static pressure gradients and occurs at both operating conditions.

- The back-flow swirls inherently due to the rotation of the impeller. This induces a swirl on the incoming flow being entrained into the impeller. Thereby, the incidence angle and efficiency of the blades are significantly influenced.

- An enhanced amount of flow recirculation from the end of the volute into the initial part of the volute occurs for off-design operating conditions. The formation of a separation bubble under the tongue is induced by this flow recirculation.

- Only at surge, global flow oscillation in form of a limit cycle at a distinct frequency occurs. A high pressure gradient downstream of the impeller induces back-flow over the impeller and lowers the blade efficiency, which leads to less fluid moved downstream (emptying) and a reduction of the pressure gradient. Due to the reduced pressure gradient, less back-flow is forced upstream over the impeller tips and the blade efficiency is increased, which causes more fluid to be moved downstream (filling) and the pressure gradient downstream of the impeller increases again.

- Therefore, inlet swirl counteracting and stabilising the effect of the back-flow swirl can be efficiently used to extend the surge margins.

\section{Acknowledgements}

The authors are grateful to acknowledge Prof. Ephraim Gutmark and Dr. Matthieu Gancedo at University of Cincinnati for providing the experimental data used in this study. Further, the authors would like to thank Jyothish Kumar Venkataramanan for setting up the preliminary simulations leading to this publication. This work was supported by the Competence Center for 
Gas Exchange (CCGEx), Volvo Cars, Volvo GTT, SCANIA and the Swedish Energy Agency (STEM). The computational resources provided through the Swedish National Infrastructure for Computing via PDC (SNIC 2013/1-72) are greatly acknowledged.

\section{References}

[1] X. Wang, Y. Ge, L. Yu, X. Feng, Effects of altitude on the thermal efficiency of a heavy-duty diesel engine, Energy 59 (2013) 543-548.

[2] R. H. Aungier, Centrifugal compressors: a strategy for aerodynamic design and analysis, American Society of Mechanical Engineers (ASME Press, New York, USA), 2000.

[3] J. Andersen, F. Lindström, F. Westin, Surge definitions for radial compressors in automotive turbochargers, SAE International Journal of Engines 1 (1) (2008) 218

[4] D. Fink, N. Cumpsty, E. Greitzer, Surge dynamics in a freespool centrifugal compressor system, Journal of Turbomachinery 114 (2) (1992) 321-332.

[5] J. Galindo, J. Serrano, H. Climent, A. Tiseira, Experiments and modelling of surge in small centrifugal compressor for automotive engines, Experimental Thermal and Fluid Science 32 (3) (2008) 818-826.

[6] M. Yang, R. Martinez-Botas, Y. Zhang, X. Zheng, Effect of selfrecirculation-casing treatment on high pressure ratio centrifugal compressor, Journal of Propulsion and Power (2016) 1-9.

[7] J. Galindo, J. Serrano, X. Margot, A. Tiseira, N. Schorn, H. Kindl, Potential of flow pre-whirl at the compressor inlet of automotive engine turbochargers to enlarge surge margin and overcome packaging limitations, International journal of heat and fluid flow 28 (3) (2007) 374-387.

[8] N. Figurella, R. Dehner, A. Selamet, K. Miazgowicz, A. Karim, R. Host, Effect of aerodynamically induced pre-swirl on centrifugal compressor acoustics and performance, SAE International Journal of Passenger Cars-Mechanical Systems 8 (3) (2015) 995-1002.

[9] E. Sundström, B. Semlitsch, M. Mihaescu, Similarities and differences concerning flow characteristics in centrifugal compressors of different size, in: International Conference on Jets, Wakes and Separated Flows (ICJWSF2015), 2015

[10] G. Després, G. N. Boum, F. Leboeuf, D. Chalet, P. Chesse, A. Lefebvre, Simulation of near surge instabilities onset in a turbocharger compressor, Proceedings of the Institution of $\mathrm{Me}$ chanical Engineers, Part A: Journal of Power and Energy 227 (6) (2013) 665-673.

[11] J. A. Bourgeois, J. C. Nichols, G. H. Watson, R. J. Martinuzzi, Single passage detached eddy simulation of a centrifugal compressor stage using the time transformation method, in: ASME Turbo Expo 2015: Turbine Technical Conference and Exposition, American Society of Mechanical Engineers, 2015, pp. V02BT39A042-V02BT39A042.

[12] B. Semlitsch, V. JyothishKumar, M. Mihaescu, L. Fuchs, E. Gutmark, M. Gancedo, Numerical flow analysis of a centrifugal compressor with ported and without ported shroud, SAE Technical Paper 2014-01-1655.

[13] B. Semlitsch, V. Jyothishkumar, M. Mihaescu, L. Fuchs, E. J. Gutmark, Investigation of the surge phenomena in a centrifugal compressor using large eddy simulation, in: ASME 2013 International Mechanical Engineering Congress and Exposition, no. IMECE2013-66301, 2013, p. 10 pages.

[14] F. Mendonça, O. Baris, G. Capon, Simulation of radial compressor aeroacoustics using cfd, in: ASME Turbo Expo 2012:
Turbine Technical Conference and Exposition, American Society of Mechanical Engineers, 2012, pp. 1823-1832.

[15] N. Gourdain, F. Sicot, F. Duchaine, L. Gicquel, Large eddy simulation of flows in industrial compressors: a path from 2015 to 2035, Philosophical Transactions of the Royal Society A: Mathematical, Physical and Engineering Sciences 372 (2022) (2014) 20130323.

[16] K. Nakonieczny, Entropy generation in a diesel engine turbocharging system, Energy 27 (11) (2002) 1027-1056.

[17] M. Gancedo, E. Gutmark, E. Guillou, Piv measurements of the flow at the inlet of a turbocharger centrifugal compressor with recirculation casing treatment near the inducer, Experiments in Fluids 57 (2) (2016) 1-19.

[18] E. Sundström, B. Semlitsch, M. Mihaescu, Assessment of the 3d flow in a centrifugal compressor using steady-state and unsteady flow solvers, SAE Technical Paper 2014-01-2856.

[19] J. Smagorinsky, General circulation experiments with the primitive equations: I. the basic experiment*, Monthly weather review 91 (3) (1963) 99-164.

[20] A. Kravchenko, P. Moin, On the effect of numerical errors in large eddy simulations of turbulent flows, Journal of Computational Physics 131 (2) (1997) 310-322.

[21] S. Ghosal, An analysis of numerical errors in large-eddy simulations of turbulence, Journal of Computational Physics 125 (1) (1996) 187-206.

[22] L. G. Margolin, W. J. Rider, A rationale for implicit turbulence modelling, International Journal for Numerical Methods in Fluids 39 (9) (2002) 821-841.

[23] L. G. Margolin, W. J. Rider, F. F. Grinstein, Modeling turbulent flow with implicit les, Journal of Turbulence 7 (15) (2006) 1-27.

[24] C. Fureby, F. F. Grinstein, Large eddy simulation of highreynolds-number free and wall-bounded flows, Journal of Computational Physics 181 (1) (2002) 68 - 97.

[25] Z. Sun, C. Tan, D. Zhang, Flow field structures of the impeller backside cavity and its influences on the centrifugal compressor, in: ASME Turbo Expo 2009: Power for Land, Sea, and Air, American Society of Mechanical Engineers, 2009, pp. 13491360.

[26] K. Guleren, I. Afgan, A. Turan, Predictions of turbulent flow for the impeller of a nasa low-speed centrifugal compressor, Journal of Turbomachinery 132 (2) (2010) 021005.

[27] P. J. Schmid, Dynamic mode decomposition of numerical and experimental data, J. Fluid Mech. 656 (2010) 5-28.

[28] T. Raitor, W. Neise, Sound generation in centrifugal compressors, Journal of Sound and Vibration 314 (3) (2008) 738-756. 